\title{
RELATIVE PRESSURE, RELATIVE EQUILIBRIUM STATES, COMPENSATION FUNCTIONS AND MANY-TO-ONE CODES BETWEEN SUBSHIFTS
}

\author{
PETER WALTERS
}

\begin{abstract}
Let $S: X \rightarrow X, T: Y \rightarrow Y$ be continuous maps of compact metrizable spaces, and let $\pi: X \rightarrow Y$ be a continuous surjection with $\pi \circ S=$ $T \circ \pi$. We investigate the notion of relative pressure, which was introduced by Ledrappier and Walters, and study some maximal relative pressure functions that tie in with relative equilibrium states. These ideas are connected with the notion of compensation function, first considered by Boyle and Tuncel, and we show that a compensation function always exists when $S$ and $T$ are subshifts. A function $F \in C(X)$ is a compensation function if $P(S, F+\phi \circ \pi)=$ $P(T, \phi) \forall \phi \in C(Y)$. When $S$ and $T$ are topologically mixing subshifts of finite type, we relate compensation functions to lifting $T$-invariant measures to $S$-invariant measures, obtaining some results of Boyle and Tuncel. We use compensation functions to describe different types of quotient maps $\pi$. An example is given where no compensation function exists.
\end{abstract}

0. Introduction. When $S: X \rightarrow X, T: Y \rightarrow Y$ are continuous maps of compact metrizable spaces and $\pi: X \rightarrow Y$ is a continuous surjection with $\pi \circ S=T \circ$ $\pi$, the concept of relative pressure has been defined and studied by Ledrappier and Walters $[\mathbf{L}, \mathbf{W}]$. We continue this study in $\S 1$ by considering equivalent definitions and invariance properties. In $\S 2$ we study a maximal average relative pressure function $W: C(X) \rightarrow R$, where $C(X)$ denotes the space of all real-valued continuous functions on $X$. It is a convex function, and we study its tangent functionals. These are closely related to relative equilibrium states.

A compensation function for $(S, T, \pi)$ is a function $F \in C(X)$ with

$$
P(S, F+\phi \circ \pi)=P(T, \phi) \quad \forall \phi \in C(Y),
$$

where $P(S, \cdot)$ is the pressure of $S$ and $P(T, \cdot)$ is the pressure of $T$. Boyle and Tuncel have shown the importance of such functions $[\mathbf{B}, \mathbf{T}]$. In $\S 3$ we show that a compensation function always exists when $S$ and $T$ are subshifts. We also obtain equivalent conditions for a function to be a compensation function.

For a topologically mixing subshift of finite type $S: X \rightarrow X$ there is an extensive theory of equilibrium states: in particular, many functions have unique equilibrium states. In $\S 4$ we consider a class $\mathcal{F}(X)$ of such functions, and when $S$ and $T$ are both topologically mixing subshifts of finite type, we connect the idea of compensation function to that of lifting equilibrium states of functions in $\mathcal{F}(Y)$ to equilibrium states of functions in $\mathcal{F}(X)$. In particular, if the unique measure of maximal entropy for $T$ lifts to the equilibrium state of a function $f \in \mathcal{F}(X)$ then $f+c$ is

Received by the editors July 2, 1985.

1980 Mathematics Subject Classification. Primary 28D99, 58F11; Secondary 54H20, 60J10. code.

Key words and phrases. Pressure, compensation function, equilibrium state, Markov measure, 
a compensation function for some $c \in R$. One can characterise different kinds of quotient maps $\pi$ by the type of compensation functions they admit, and we give examples of some of these types. The example of Marcus, Petersen and Williams $[\mathbf{M}, \mathbf{P}, \mathbf{W}]$ is shown to have no compensation function in $\mathcal{F}(X)$.

In $\S 5$ we give an example where no compensation function exists and $S, T$ are topologically transitive homeomorphisms.

We shall use notation from $\left[\mathbf{W}_{\mathbf{3}}\right]$. If $S: X \rightarrow X$ is a continous map of a compact metrizable space then $M(X, S)$ will denote the space of all $S$-invariant Borel probability measures on $X$. A Borel subset $B$ of $X$ will be called a total probability set for $S$ if $\mu(B)=1 \forall \mu \in M(X, S)$. If $\mu \in M(X, S)$ then $h_{\mu}(S)$ will denote the measure-theoretic entropy of $S$ relative to $\mu$. The topological entropy of $S$ will be denoted by $h(S)$. We use $C(X)$ for the space of real-valued continuous functions on $X$, and $\|f\|$ denotes the supremum norm of $f \in C(X)$. The pressure of $S$ evaluated at $f \in C(X)$ is denoted $P(S, f)$. All logarithms used are natural logarithms. Recall that $\mu \in M(X, S)$ is an equilibrium state of $f \in C(X)$ if $h_{\mu}(S)+\int f d \mu=P(S, f)$.

When $S: X \rightarrow X, T: Y \rightarrow Y$ are continuous maps of compact metrizable spaces and $\pi: X \rightarrow Y$ is a continuous surjection with $\pi \circ S=T \circ \pi$, we shall use relative entropy $h_{\mu}\left(\left.S\right|_{T}\right), \mu \in M(X, S)$, defined as follows $[\mathbf{A}, \mathbf{R}]$. Let

$$
h_{\mu}\left(\left.S\right|_{T}\right)=\sup \left\{h_{\mu}\left(\left.S\right|_{T}, \xi\right) \mid \xi \text { is a finite partition of } X\right\}
$$

and

$$
h_{\mu}\left(\left.S\right|_{T}, \xi\right)=\lim _{n \rightarrow \infty} \frac{1}{n} H_{\mu}\left(\left(\bigvee_{i=0}^{n-1} S^{-i} \xi\right) / \pi^{-1} \varepsilon_{Y}\right),
$$

where $\varepsilon_{Y}$ is the partition of $Y$ into points. We have

$$
h_{\mu}(S)=h_{\mu \circ \pi^{-1}}(T)+h_{\mu}\left(\left.S\right|_{T}\right), \quad \mu \in M(X, S)
$$

$[\mathbf{A}, \mathbf{R}]$. It can happen that $h_{\mu}(S)$ and $h_{\mu \circ \pi^{-1}}(T)$ are both infinite but $h_{\mu}\left(\left.S\right|_{T}\right)$ is finite, so in these cases consideration of $h_{\mu}\left(\left.S\right|_{T}\right)$ makes sense whereas $h_{\mu}(S)-$ $h_{\mu \circ \pi^{-1}}(T)$ does not. However, when $h_{\mu}(S)<\infty, h_{\mu}\left(\left.S\right|_{T}\right)=h_{\mu}(S)-h_{\mu \circ \pi^{-1}}(T)$.

Throughout the paper $S: X \rightarrow X, T: Y \rightarrow Y$ denote continuous maps of compact metrizable spaces, and $\pi: X \rightarrow Y$ denotes a continous surjection with $\pi S=T \pi$. In some sections we make additional assumptions, which are stated at the beginning of the sections. Members of $C(Y)$ will normally be denoted by Greek letters $\phi, \psi$, etc., and members of $C(X)$ by $f, g, h$, etc. For $f \in C(X),\left(S_{n} f\right)(x)$ denotes $\sum_{i=0}^{n-1} f\left(S^{i} x\right)$, and for $\phi \in C(Y),\left(T_{n} \phi\right)(y)$ denotes $\sum_{i=0}^{n-1} \phi\left(T^{i} y\right)$.

1. Relative pressure. The idea of relative pressure was introduced in $[\mathbf{L}, \mathbf{W}]$. Let $X, Y$ be two compact metric spaces, and let $S: X \rightarrow X, T: Y \rightarrow Y$ and $\pi: X \rightarrow Y$ be continuous maps such that $\pi$ is surjective and $\pi S=T \pi$. (We have changed the notation of $[\mathbf{L}, \mathbf{W}]$.) We say $T$ is a factor of $S$ by the map $\pi$, or that $\pi$ is a semiconjugacy from $S$ to $T$. We now define relative pressure. For each $f \in C(X)$ we shall obtain a map $P(S, \pi, f): Y \rightarrow R$, which may not be continuous, and $P(S, \pi, f)(y)$ will measure the relative pressure of $f$ over $y \in Y$. Choose any metric $d$ on $X$. Recall that for $n \geq 1$ and $\delta>0$ a finite subset $E$ of $X$ is $(n, \delta)$ separated with respect to $S$ if $x, y \in E, x \neq y$, imply $\max _{0 \leq i \leq n-1} d\left(S^{i} x, S^{i} y\right)>\delta$. 
For $y \in Y$ let

$$
\begin{aligned}
& P_{n}(S, \pi, f, \delta)(y) \\
& \quad=\sup \left\{\sum_{x \in E} \exp \left(\left(S_{n} f\right)(x)\right) \mid E \text { is }(n, \delta) \text { separated and } E \subset \pi^{-1}(y)\right\} .
\end{aligned}
$$

Now let

$$
P(S, \pi, f)(y)=\lim _{\delta \rightarrow 0} \limsup _{n \rightarrow \infty} \frac{1}{n} \log P_{n}(S, \pi, f, \delta)(y) .
$$

Although $P_{n}$ depends on the metric $d$, the quantity $P(S, \pi, f)(y)$ is independent of the metric chosen.

Let $M(X, S)$ denote the space of all $S$-invariant Borel probability measures on $X$, and let $M(Y, T)$ denote the corresponding space for $T$. It was shown in $[\mathbf{L}, \mathbf{W}]$ that $P(S, \pi, f): Y \rightarrow R$ is Borel measurable for all $f \in C(X)$, and for each $m \in M(Y, T)$ and $f \in C(X)$,

$$
\int P(S, \pi, f) d m=\sup \left\{h_{\mu}\left(\left.S\right|_{T}\right)+\int f d \mu \mid \mu \in M(X, S) \text { and } \mu \circ \pi^{-1}=m\right\} .
$$

We shall call this result the relative variational principle. (It reduces to the usual variational principle when $Y$ is a space with only one point.)

Throughout this section the maps $S, T, \pi$ will be as described above. We shall assume $P(S, \pi, f)(y)$ never takes the value $\infty$, for each $f \in C(X)$; by Proposition 1.1 this is equivalent to the function $P(S, \pi, 0)(y)$ never taking the value $\infty$. This assumption holds when $h(S)<\infty$. There are the following elementary properties which are proved in the same way as the corresponding statements for pressure. (See $\left[\mathbf{W}_{3}\right.$, p. 214].)

Proposition 1.1. If $f, g \in C(X), y \in Y$ and $c \in R$, then

(i) $f \leq g$ implies $P(S, \pi, f)(y) \leq P(S, \pi, g)(y)$,

(ii) $|P(S, \pi, f)(y)-P(S, \pi, g)(y)| \leq\|f-g\|$,

(iii) $P(S, \pi, \cdot)(y)$ is a convex, real-valued function on $C(X)$,

(iv) $P(S, \pi, f+c)(y)=P(S, \pi, f)(y)+c$,

(v) $P(S, \pi, f+g \circ S-g)(y)=P(S, \pi, f)(y)$.

As in the case of (nonrelative) pressure there is a definition of relative pressure using spanning sets rather than separated sets. One says that a subset $F$ of $X$ $(n, \delta)$-spans $\pi^{-1}(y)$ if $\forall x \in \pi^{-1}(y) \exists z \in F$ such that $\max _{0 \leq i \leq n-1} d\left(S^{i} x, S^{i} z\right) \leq \delta$. For $f \in C(X)$ let

$$
\begin{aligned}
& Q_{n}(S, \pi, f, \delta)(y)=\inf \left\{\sum_{x \in F} \exp \left(\left(S_{n} f\right)(x)\right) \mid\right. \\
&\left.F(n, \delta) \text {-spans } \pi^{-1}(y) \text { and } F \subset \pi^{-1}(y)\right\} .
\end{aligned}
$$

Proposition 1.2. For each $f \in C(X)$ and $y \in Y$,

$$
P(S, \pi, f)(y)=\lim _{\delta \rightarrow 0} \limsup _{n \rightarrow \infty} \frac{1}{n} \log Q_{n}(S, \pi, f, \delta)(y) .
$$


PROOF. In the definition of $P_{n}(S, \pi, f, \delta)(y)$ it suffices to take the supremum over the collection of those $(n, \delta)$-separated sets which are maximal (in the sense that the addition of any point of $\pi^{-1}(y)$ forces the new set to not be $(n, \delta)$-separated). Such a maximal $(n, \delta)$-separated subset of $\pi^{-1}(y)$ is a set which $(n, \delta)$-spans $\pi^{-1}(y)$, so $Q_{n}(S, \pi, f, \delta)(y) \leq P_{n}(S, \pi, f, \delta)(y)$. Therefore

$$
\lim _{\delta \rightarrow 0} \limsup _{n \rightarrow \infty} \frac{1}{n} \log Q_{n}(S, \pi, f, \delta)(y) \leq P(S, \pi, f)(y) .
$$

For $\delta>0$ let

$$
v(f, \delta / 2)=\sup \left\{\left|f(x)-f\left(x^{\prime}\right)\right| \mid d\left(x, x^{\prime}\right) \leq \delta / 2\right\} .
$$

Let $E \subset \pi^{-1}(y)$ be $(n, \delta)$-separated, and let $F \subset \pi^{-1}(y)$ be a set which $(n, \delta / 2)$ spans $\pi^{-1}(y)$. Define a map $\Phi: E \rightarrow F$ by choosing $\Phi(x) \in F$ so that

$$
\max _{0 \leq i \leq n-1} d\left(S^{i} x, S^{i} \Phi(x)\right) \leq \delta / 2 .
$$

The fact that $E$ is $(n, \delta)$-separated implies that $\Phi$ is one-to-one. Therefore

$$
\begin{aligned}
\sum_{z \in F} \exp \left(\left(S_{n} f\right)(z)\right) & \geq \sum_{x \in E} \exp \left(\left(S_{n} f\right)(\Phi(x))\right) \\
& \geq \exp (-n v(f, \delta / 2)) \sum_{x \in E} \exp \left(\left(S_{n} f\right)(x)\right)
\end{aligned}
$$

Hence

$$
Q_{n}(S, \pi, f, \delta / 2)(y) \geq \exp (-n v(f, \delta / 2)) P_{n}(S, \pi, f, \delta)(y)
$$

Since $v(f, \delta / 2)$ goes to zero as $\delta$ goes to zero, this finishes the proof.

Note that, by the first part of the proof, we always have

$$
Q_{n}(S, \pi, f, \delta)(y) \leq P_{n}(S, \pi, f, \delta)(y) .
$$

The above proof also shows that if we define $\tilde{Q}_{n}(S, \pi, f, \delta)(y)$ to be

$$
\inf \left\{\sum_{x \in F} \exp \left(\left(S_{n} f\right)(x)\right) \mid F(n, \delta) \text {-spans } \pi^{-1}(y)\right\}
$$

we get

$$
P(S, \pi, f)(y)=\lim _{\delta \rightarrow 0} \limsup _{n \rightarrow \infty} \frac{1}{n} \log \tilde{Q}_{n}(S, \pi, f, \delta)(y) .
$$

We can also define relative pressure by using open covers. If $\gamma$ is an open cover of $X$ and $X_{1}$ is a subset of $X$, we let $\left.\gamma\right|_{X_{1}}$ denote the cover of $X_{1}$ by the sets $C \cap X_{1}, C \in \gamma$. For an open cover $\alpha$ of $X, f \in C(X), y \in Y$ and $n \geq 1$, let

$$
q_{n}(S, \pi, f, \alpha)(y)=\inf \left\{\sum_{B \in \beta} \inf _{x \in B} \exp \left(\left(S_{n} f\right)(x)\right) \mid\right.
$$

$$
\left.\beta \text { is a finite subcover of }\left.\left(\bigvee_{i=0}^{n-1} S^{-i} \alpha\right)\right|_{\pi^{-1}(y)}\right\}
$$


and

$$
\begin{aligned}
p_{n}(S, \pi, f, \alpha)(y)=\inf \left\{\sum_{B \in \beta} \sup _{x \in B} \exp \left(\left(S_{n} f\right)(x)\right) \mid\right. \\
\left.\beta \text { is a finite subcover of }\left.\left(\bigvee_{i=0}^{n-1} S^{-i} \alpha\right)\right|_{\pi^{-1}(y)}\right\} .
\end{aligned}
$$

Clearly $q_{n}(S, \pi, f, \alpha)(y) \leq p_{n}(S, \pi, f, \alpha)(y)$.

We have the following connections with $Q_{n}$ and $P_{n}$. Recall that the diameter, $\operatorname{diam}(\alpha)$, of a cover $\alpha$ is the supremum of the diameters of all the members of the cover $\alpha$.

LEMMA 1.1. (i) If $\alpha$ is an open cover of $X$ with Lebesgue number $\delta$, then

$$
q_{n}(S, \pi, f, \alpha)(y) \leq Q_{n}(S, \pi, f, \delta / 2)(y) .
$$

(ii) If $\varepsilon>0$ and $\gamma$ is an open cover of $X$ with $\operatorname{diam}(\gamma) \leq \varepsilon$, then

$$
P_{n}(S, \pi, f, \varepsilon)(y) \leq p_{n}(S, \pi, f, \gamma)(y)
$$

ProOF. We shall use $\bar{B}(x ; \varepsilon)$ to denote the closed ball in $X$ of radius $\varepsilon$.

(i) If $F$ is a subset of $\pi^{-1}(y)$ which $(n, \delta / 2)$-spans $\pi^{-1}(y)$, then

$$
\pi^{-1}(y) \subset \bigcup_{x \in F} \bigcap_{i=0}^{n-1} S^{-i} \bar{B}\left(S^{i} x ; \delta / 2\right) .
$$

Therefore

$$
q_{n}(S, \pi, f, \alpha)(y) \leq \sum_{x \in F} \exp \left(\left(S_{n} f\right)(x)\right)
$$

Hence

$$
q_{n}(S, \pi, f, \alpha)(y) \leq Q_{n}(S, \pi, f, \delta / 2)(y)
$$

(ii) Let $E$ be an $(n, \varepsilon)$-separated subset of $\pi^{-1}(y)$. No member of $\bigvee_{i=0}^{n-1} S^{-i} \gamma$ contains more than one element of $E$, so we have

$$
\sum_{x \in E} \exp \left(\left(S_{n} f\right)(x)\right) \leq p_{n}(S, \pi, f, \gamma)(y)
$$

Therefore

$$
P_{n}(S, \pi, f, \varepsilon)(y) \leq p_{n}(S, \pi, f, \gamma)(y) .
$$

The following result helps to calculate relative pressure.

Proposition 1.3. For any $f \in C(X), y \in Y$ and any sequence $\left\{\alpha_{k}\right\}_{1}^{\infty}$ of open covers of $X$ with $\operatorname{diam}\left(\alpha_{k}\right) \rightarrow 0$, we have

$$
\begin{aligned}
P(S, \pi, f)(y) & =\lim _{k \rightarrow \infty} \limsup _{n \rightarrow \infty} \frac{1}{n} \log p_{n}\left(S, \pi, f, \alpha_{k}\right)(y) \\
& =\lim _{k \rightarrow \infty} \limsup _{n \rightarrow \infty} \frac{1}{n} \log q_{n}\left(S, \pi, f, \alpha_{k}\right)(y) .
\end{aligned}
$$

PROOF. If $\alpha$ is an open cover of $X$ of diameter $\delta$ and

$$
v(f, \delta)=\sup \left\{\left|f(x)-f\left(x^{\prime}\right)\right| \mid d\left(x, x^{\prime}\right) \leq \delta\right\},
$$


then

$$
p_{n}(S, \pi, f, \alpha)(y) \leq e^{n v(f, \delta)} q_{n}(S, \pi, f, \alpha)(y) \quad \forall y \in Y .
$$

Let $\delta_{k}=\operatorname{diam}\left(\alpha_{k}\right)$. Then $P_{n}\left(S, \pi, f, \delta_{k}\right)(y) \leq p_{n}\left(S, \pi, f, \alpha_{k}\right)(y)$ by Lemma 1.1(ii). Therefore

$$
\begin{aligned}
P(S, \pi, f)(y) & \leq \liminf _{k \rightarrow \infty} \limsup _{n \rightarrow \infty} \frac{1}{n} \log p_{n}\left(S, \pi, f, \alpha_{k}\right)(y) \\
& \leq \liminf _{k \rightarrow \infty} \limsup _{n \rightarrow \infty} \frac{1}{n} \log q_{n}\left(S, \pi, f, \alpha_{k}\right)(y) .
\end{aligned}
$$

Let $\varepsilon_{k}$ be a Lebesgue number for $\alpha_{k}$. By Lemma 1.1(i) we have $q_{n}\left(S, \pi, f, \alpha_{k}\right)(y) \leq$ $P_{n}\left(S, \pi, f, \varepsilon_{k} / 2\right)(y)$. Therefore

$$
e^{-n v\left(f, \delta_{k}\right)} p_{n}\left(S, \pi, f, \alpha_{k}\right)(y) \leq q_{n}\left(S, \pi, f, \alpha_{k}\right)(y) \leq P_{n}\left(S, \pi, f, \varepsilon_{k} / 2\right)(y)
$$

and

$$
\begin{aligned}
-v\left(f, \delta_{k}\right)+\limsup _{n \rightarrow \infty} \frac{1}{n} \log p_{n}\left(S, \pi, f, \alpha_{k}\right)(y) & \leq \limsup _{n \rightarrow \infty} \frac{1}{n} \log q_{n}\left(S, \pi, f, \alpha_{k}\right)(y) \\
& \leq P(S, \pi, f)(y) .
\end{aligned}
$$

Therefore

$$
\begin{aligned}
\limsup _{k \rightarrow \infty} \limsup _{n \rightarrow \infty} \frac{1}{n} \log p_{n}\left(S, \pi, f, \alpha_{k}\right)(y) & \leq \limsup _{k \rightarrow \infty} \limsup _{n \rightarrow \infty} \frac{1}{n} \log q_{n}\left(S, \pi, f, \alpha_{k}\right)(y) \\
& \leq P(S, \pi, f)(y)
\end{aligned}
$$

so that

$$
\lim _{k \rightarrow \infty} \limsup _{n \rightarrow \infty} \frac{1}{n} \log p_{n}\left(S, \pi, f, \alpha_{k}\right)(y)
$$

and

$$
\lim _{k \rightarrow \infty} \limsup _{n \rightarrow \infty} \frac{1}{n} \log q_{n}\left(S, \pi, f, \alpha_{k}\right)(y)
$$

exist and equal $P(S, \pi, f)(y)$.

Recall that we are calling (following Mañé) a Borel subset $B$ of $Y$ a total probability set for $T$ if $m(B)=1 \forall m \in M(Y, T)$.

LEMMA 1.2. For each open cover $\alpha$ and each $f \in C(X)$ the set

$$
\left\{y \in Y \mid \lim _{n \rightarrow \infty} \frac{1}{n} \log p_{n}(S, \pi, f, \alpha)(y) \text { exists }\right\}
$$

is a total probability set for $T$.

ProOF. We have, for all $n, k \geq 1$,

$$
p_{n+k}(S, \pi, f, \alpha)(y) \leq p_{n}(S, \pi, f, \alpha)(y) \cdot p_{k}(S, \pi, f, \alpha)\left(T^{n} y\right),
$$

so the subadditive ergodic theorem gives the result.

COROLlaRY 1.1. For a sequence $\left\{\alpha_{k}\right\}_{1}^{\infty}$ of open covers of $X$ with $\operatorname{diam}\left(\alpha_{k}\right) \rightarrow$ 0 , the set of all points $y \in Y$ at which

$$
\begin{aligned}
P(S, \pi, f)(y) & =\lim _{k \rightarrow \infty} \lim _{n \rightarrow \infty} \frac{1}{n} \log p_{n}\left(S, \pi, f, \alpha_{k}\right)(y) \\
& =\lim _{k \rightarrow \infty} \liminf _{n \rightarrow \infty} \frac{1}{n} \log q_{n}\left(S, \pi, f, \alpha_{k}\right)(y)
\end{aligned}
$$

for all $f \in C(X)$ is a total probability set. 
Proof. Since $C(X)$ is separable, it is enough to consider the set $Y_{f}$ where the above equalities hold for a particular $f \in C(X)$. Using the notation of the proof of Proposition 1.3, we have

$$
e^{-n v\left(f, \delta_{k}\right)} p_{n}\left(S, \pi, f, \alpha_{k}\right)(y) \leq q_{n}\left(S, \pi, f, \alpha_{k}\right)(y) \leq p_{n}\left(S, \pi, f, \alpha_{k}\right)(y) .
$$

The result follows from Lemma 1.2 and Proposition 1.3.

Proposition 1.4. Let $Y_{0}$ consist of all points $y \in Y$ for which, $\forall f \in C(X)$,

$$
\begin{aligned}
P(S, \pi, f)(y) & =\lim _{\delta \rightarrow 0} \liminf _{n \rightarrow \infty} \frac{1}{n} Q_{n}(S, \pi, f, \delta)(y) \\
& =\lim _{\delta \rightarrow 0} \liminf _{n \rightarrow \infty} \frac{1}{n} \log P_{n}(S, \pi, f, \delta)(y) .
\end{aligned}
$$

Then $Y_{0}$ is a total probability set for $T$.

PrOOF. It suffices to show that for each $f \in C(X)$ the set $Y_{f}$ of points $y$ where the expressions agree is a total probability set.

Let $\alpha_{k}$ be the cover of $X$ by all open balls of radius $3 / k$, and let $\gamma_{k}$ be any cover of $X$ by open balls of radius $1 / 2 k$.

If $v(f, \varepsilon)=\sup \left\{\left|f(x)-f\left(x^{\prime}\right)\right| \mid d\left(x, x^{\prime}\right) \leq \varepsilon\right\}$, then we easily get

$$
e^{-n v(f, 6 / k)} p_{n}\left(S, \pi, f, \alpha_{k}\right)(y) \leq q_{n}\left(S, \pi, f, \alpha_{k}\right)(y),
$$

so that, by Lemma 1.1,

$$
\begin{aligned}
& e^{-n v(f, 6 / k)} p_{n}\left(S, \pi, f, \alpha_{k}\right)(y) \\
& \quad \leq q_{n}\left(S, \pi, f, \alpha_{k}\right)(y) \leq Q_{n}(S, \pi, f, 1 / k)(y) \\
& \quad \leq P_{n}(S, \pi, f, 1 / k)(y) \leq p_{n}\left(S, \pi, f, \gamma_{k}\right)(y) .
\end{aligned}
$$

By Lemma 1.2, $\left\{y \in Y \mid \lim _{n \rightarrow \infty} n^{-1} \log p_{n}\left(S, \pi, f, \alpha_{k}\right)(y)\right.$ exists $\}$ is a total probability set $Y_{k}$ for each $k$. At points of $\bigcap_{k=1}^{\infty} Y_{k}$ we can first take $\lim \sup _{n \rightarrow \infty}$ in the above string of inequalities and then $\liminf _{n \rightarrow \infty}$ and use Corollary 1.1.

We note that in the conclusion of Proposition 1.4 the set $Y_{0}$ can be different from $Y$. The reason is that if $y$ is a point in $Y_{0}$ and we put $f=\phi \circ \pi$ for $\phi \in C(Y)$, then $\lim _{n \rightarrow \infty} n^{-1} \sum_{i=0}^{n-1} \phi\left(T^{i} y\right)$ exists.

We now consider invariance properties of relative pressure.

Proposition 1.5. For each $f \in C(X)$ and $y \in Y$ we have $P(S, \pi \circ S, f)(y)=$ $P(S, \pi, f)(y)$.

PROOF. The fibre of $\pi \circ S$ over $y$ is the set $(\pi \circ S)^{-1}(y)=S^{-1} \pi^{-1}(y)$. Let $E$ be an $(n, \varepsilon)$-separated subset of $\pi^{-1}(y)$. For each $x \in E$ choose one point from $S^{-1} x$ and let $E^{\prime}$ denote the collection of these choices. Then $E^{\prime}$ is an $(n+1, \varepsilon)$-separated subset of $(\pi \circ S)^{-1}(y)$. Therefore

$$
P_{n}(S, \pi, f, \varepsilon)(y) \leq P_{n+1}(S, \pi \circ S, f, \varepsilon)(y) e^{\|f\|},
$$

and hence

$$
P(S, \pi, f)(y) \leq P(S, \pi \circ S, f)(y) .
$$

Now suppose $B$ is an $(n, \varepsilon)$-separated subset of $S^{-1} \pi^{-1}(y)$. Then $S B \subset \pi^{-1}(y)$. Let $A$ denote a maximal $(n-1, \varepsilon / 2)$-separated subset of $S B$. For each $a \in A$ choose 
some $b \in B \cap S^{-1} a$. Let $\left\{b_{1}, \ldots, b_{k_{n}}\right\}$ be the points chosen in this way. Then $A=\left\{S b_{1}, \ldots, S b_{k_{n}}\right\}$. Let $b \in B$. Either $S b \in A$ and therefore $S b=S b_{i}$ for some $i$, or $S b \neq A$ and there exists $b_{i}$ with $\max _{0 \leq j \leq n-2} d\left(S^{j} S b, S^{j} S b_{i}\right) \leq \varepsilon / 2$ since $A$ was chosen to be maximal. So for each $b \in B$ there is some $b_{i}$ with $d\left(S^{j} b, S^{j} b_{i}\right) \leq \varepsilon / 2$ for $1 \leq j \leq n-1$. Let $d(\varepsilon)$ denote the maximum number of $\varepsilon$-separated points in $X$. For each $b_{i}$ the set $\left\{b \in B \mid d\left(S^{j} b, S^{j} b_{i}\right) \leq \varepsilon / 2,1 \leq j \leq n-1\right\}$ is $\varepsilon$-separated and so consists of at most $d(\varepsilon)$ points. If

$$
v(f, \varepsilon)=\sup \left\{\left|f(x)-f\left(x^{\prime}\right)\right| \mid d\left(x, x^{\prime}\right) \leq \varepsilon\right\},
$$

then

$$
\sum_{x \in B} \exp \left(S_{n} f\right)(x) \leq d(\varepsilon) \sum_{z \in A} \exp \left(S_{n-1} f\right)(z) \exp (\|f\|+(n-1) v(f, \varepsilon)) .
$$

Therefore

$$
P_{n}(S, \pi \circ S, f, \varepsilon)(y) \leq d(\varepsilon) \exp (\|f\|+(n-1) v(f, \varepsilon)) P_{n-1}(S, \pi, f, \varepsilon / 2)(y)
$$

and hence

$$
P(S, \pi \circ S, f)(y) \leq P(S, \pi, f)(y) .
$$

We clearly also have $P(S, \pi, f \circ S)(y)=P(S, \pi, f)(y) \forall y \in Y, \forall f \in C(X)$.

Proposition 1.6. For each $f \in C(X)$ and each $y \in Y$ we have

$$
P(S, \pi, f)(y) \leq P(S, \pi, f)(T y)
$$

PrOOF. Since $(\pi \circ S)^{-1}(T y)=\pi^{-1} T^{-1} T y \supset \pi^{-1} y$, we clearly have

$$
P(S, \pi \circ S, f)(T y) \geq P(S, \pi, f)(y) .
$$

The result follows from Proposition 1.5

COROllaRY 1.2. The set $\{y \in Y \mid P(S, \pi, f)(T y)=P(S, \pi, f)(y) \forall f \in C(X)\}$ is a total probability set for $T$. Hence $P(S, \pi, f)(y)$ is constant a.e. with respect to each ergodic measure.

One can construct examples where $P(S, \pi, f)(T y) \neq P(S, \pi, f)(y)$ for some points $y$, but we do have the following result.

Proposition 1.7. Suppose that $y \in Y$ is such that $S$ maps $\pi^{-1}(y)$ onto $\pi^{-1}(T y)$. Then $P(s, \pi, f)(T y)=P(S, \pi, f)(y)$.

PROOF. Let $E$ be an $(n, \varepsilon)$-separated subset of $\pi^{-1}(T y)$. Using the assumption on $S$, for each $x \in E$ choose some point $x^{\prime} \in S^{-1} x \cap \pi^{-1} y$. Let $E^{\prime}$ denote the collection of these points. Since $E^{\prime}$ is an $(n+1, \varepsilon)$-separated subset of $\pi^{-1}(y)$, we have

$$
P_{n}(S, \pi, f)(T y) \leq \exp (\|f\|) P_{n+1}(S, \pi, f)(y) .
$$

Therefore

$$
P(S, \pi, f)(T y) \leq P(S, \pi, f)(y) .
$$

The opposite inequality is given by Proposition 1.6.

The following result gives the behaviour of relative pressure under conjugacy. 
Proposition 1.8. For $i=1,2$ let $X_{i}, Y_{i}$ be compact metric spaces, and let $S_{i}: X_{i} \rightarrow X_{i}, T_{i}: Y_{i} \rightarrow Y_{i}, \pi_{i}: X_{i} \rightarrow Y_{i}$ be continuous and satisfy $\pi_{i}\left(X_{i}\right)=Y_{i}$ and $\pi_{i} S_{i}=T_{i} \pi_{i}$. Suppose $U: X_{1} \rightarrow X_{2}$, and $V: Y_{1} \rightarrow Y_{2}$ are homeomorphisms such that $\pi_{2} U=V \pi_{1}, U S_{1}=S_{2} U, V T_{1}=T_{2} V$. Then for each $f \in C\left(X_{1}\right)$ and $y \in Y_{1}$ we have

$$
P\left(S_{1}, \pi_{1}, f\right)(y)=P\left(S_{2}, \pi_{2}, f \circ U^{-1}\right)(V y) .
$$

The proof is an easy exercise.

In Theorem 4.6 we give a formula for $P(S, \pi, f)(y)$ when $S$ and $T$ are both subshifts.

2. Some relative pressure functions. In this section $S: X \rightarrow X, T: Y \rightarrow Y$ will again be continuous transformations of compact metric spaces and $\pi: X \rightarrow Y$ will be a continuous surjection with $\pi S=T \pi$. We shall study a maximal relative pressure function $W: C(X) \rightarrow R$ and, for each $m \in M(Y, T)$, a relative pressure function $W_{m}: C(X) \rightarrow R$.

For $f \in C(X)$ let

$$
W(S, \pi, f)=\sup \left\{\int P(S, \pi, f) d m \mid m \in M(Y, T)\right\} .
$$

By the relative variational principle we have

$$
W(S, \pi, f)=\sup \left\{h_{\mu}\left(\left.S\right|_{T}\right)+\int f d \mu \mid \mu \in M(X, S)\right\} .
$$

From now on we shall write $W(f)$ instead of $W(S, \pi, f)$. We shall assume that $W(f)$ is finite for all $f \in C(X)$. By Lemma 2.1 this is equivalent to assuming $M(0)$ is finite. Since $W(0)=\sup \left\{h_{\mu}\left(\left.S\right|_{T}\right) \mid \mu \in M(X, S)\right\}$, this assumption implies $h_{\mu}\left(\left.S\right|_{T}\right)<\infty \forall \mu \in M(X, S)$. This assumption will hold when $h(S)<\infty$. We therefore have defined a map $W: C(X) \rightarrow R$, and the elementary properties of this map are given in the following result. The proofs follow along the same lines as the corresponding properties of pressure $\left[\mathbf{W}_{3}\right.$, p. 214].

LEMMA 2.1. (i) $f \leq g$ implies $W(f) \leq W(g)$.

(ii) $W: C(X) \rightarrow R$ is continuous (in fact $|W(f)-W(g)| \leq\|f-g\|)$.

(iii) $W: C(X) \rightarrow R$ is convex.

(iv) $W(f+c)=W(f)+c \forall f \in C(X) \quad \forall c \in R$.

(v) $W(f+g \circ S-g)=W(f) \quad \forall f, g \in C(X)$.

The following lemma gives a condition under which the assumptions of Theorem 2.1 will hold.

LEMMA 2.2. If $S: X \rightarrow X$ is expansive (either an expansive homeomorphism or a positively expansive continuous map), then the relative entropy map $h\left(\left.S\right|_{T}\right)$ : $M(X, S) \rightarrow R$, defined by $\mu \rightarrow h_{\mu}\left(\left.S\right|_{T}\right)$, is upper semicontinuous.

PROOF. By considering natural extensions it suffices to consider the case when $S$ is an expansive homeomorphism and $T$ is a homeomorphism. Let $\left\{\mu_{k}\right\}_{1}^{\infty}$ be a sequence in $M(X, S)$ with $\mu_{k} \rightarrow \mu$. Let $\xi$ be a finite partition of $X$ with $\mu(\partial \xi)=0$ and such that $\xi$ is a generator with respect to each member of $M(X, S)$. (See 
Lemma 8.5(ii) and Theorem 5.25 of $\left[\mathbf{W}_{3}\right]$.) Since, for each $\nu \in M(X, S)$, we have $h_{\nu}\left(\left.S\right|_{T}\right)=h_{\nu}\left(\left.S\right|_{T}, \xi\right)$ and

$$
h_{\nu}\left(\left.S\right|_{T}, \xi\right) \leq \frac{1}{n} H_{\nu}\left(\bigvee_{i=0}^{n-1} S^{-i} \xi / \pi^{-1} \varepsilon_{Y}\right) \forall n \geq 1
$$

we can reason as follows.

$$
h_{\mu_{k}}\left(\left.S\right|_{T}\right)=h_{\mu_{k}}\left(\left.S\right|_{T}, \xi\right) \leq \frac{1}{n} H_{\mu_{k}}\left(\bigvee_{i=0}^{n-1} S^{-i} \xi / \pi^{-1} \varepsilon_{Y}\right) \quad \forall n \geq 1
$$

Now let $k \rightarrow \infty$ to give

$$
\limsup _{k \rightarrow \infty} h_{\mu_{k}}\left(\left.S\right|_{T}\right) \leq \frac{1}{n} H_{\mu}\left(\bigvee_{i=0}^{n-1} S^{-i} \xi / \pi^{-1} \varepsilon_{Y}\right)
$$

by Lemma $3.2($ ii) of $[\mathbf{L}, \mathbf{W}]$.

If we now let $n \rightarrow \infty$ we get $\lim \sup _{k \rightarrow \infty} h_{\mu_{k}}\left(\left.S\right|_{T}\right) \leq h_{\mu}\left(\left.S\right|_{T}\right)$.

The following result is a relativized version of a theorem of Ruelle ([R, p. 115]; see also [ $\mathbf{W}_{3}$, p. 222]).

THEOREM 2.1. Let $\mu_{0} \in M(X, S)$ and suppose the relative entropy map $\mu \rightarrow$ $h_{\mu}\left(\left.S\right|_{T}\right)$ is upper semicontinuous at $\mu_{0}$. Then

$$
h_{\mu_{0}}\left(\left.S\right|_{T}\right)=\inf \left\{W(g)-\int g d \mu_{0} \mid g \in C(X)\right\} .
$$

ProOF. We have

$$
W(g) \geq h_{\mu_{0}}\left(\left.S\right|_{T}\right)+\int g d \mu_{0} \quad \forall g \in C(X)
$$

so that $W(g)-\int g d \mu_{0} \geq h_{\mu_{0}}\left(\left.S\right|_{T}\right)$. Hence

$$
\inf \left\{W(g)-\int g d \mu_{0} \mid g \in C(X)\right\} \geq h_{\mu_{0}}\left(\left.S\right|_{T}\right) .
$$

To prove the converse, choose $b>h_{\mu_{0}}\left(\left.S\right|_{T}\right)$. Consider

$$
C=\left\{(\mu, t) \in M(X, S) \times R \mid 0 \leq t \leq h_{\mu}\left(\left.S\right|_{T}\right)\right\} .
$$

The set $C$ is convex by Lemma 3.2 (iii) of $[\mathbf{L}, \mathbf{W}]$. Consider $C$ as a subset of $C(X)^{*} \times$ $R$, where $C(X)^{*}$ has the weak*-topology. By the assumption on $\mu_{0}$ we have $\left(\mu_{0}, b\right) \notin$ $\bar{C}$, where $\bar{C}$ denotes the closure of $C$. Since $\bar{C}$ and $\left\{\left(\mu_{0}, b\right)\right\}$ are disjoint closed convex sets, there is a continuous linear functional $F: C(X)^{*} \times R \rightarrow R$ such that $F(\mu, t)<F\left(\mu_{0}, b\right) \forall(\mu, t) \in \bar{C}$. We know $F$ has the form $F(\mu, t)=\int f d \mu+t a$ for some $f \in C(X)$ and some $a \in R$. Therefore

$$
\int f d \mu+a h_{\mu}\left(\left.S\right|_{T}\right)<\int f d \mu_{0}+a b \quad \forall \mu \in M(X, S) .
$$

Putting $\mu=\mu_{0}$ gives $a>0$. Dividing by $a$ gives

$$
h_{\mu}\left(\left.S\right|_{T}\right)+\frac{1}{a} \int f d \mu<\frac{1}{a} \int f d \mu_{0}+b \quad \forall \mu \in M(X, S),
$$


so $W(f / a) \leq \int f / a d \mu_{0}+b$. Therefore $W(f / a)-\int f / a d \mu_{0} \leq b$, so that

$$
\inf \left\{W(g)-\int g d \mu_{0} \mid g \in C(X)\right\} \leq h_{\mu_{0}}\left(\left.S\right|_{T}\right) .
$$

Recall that a tangent functional to $W$ at $f$ is a finite signed measure $\mu: B(X) \rightarrow$ $R$ such that $W(f+g)-W(f) \geq \int g d \mu \forall g \in C(X)$. Let $W_{f}$ denote the collection of all tangent functionals to $W$ at $f$. The Hahn-Banach theorem implies that $W_{f}$ is nonempty.

THEOREM 2.2. Let $f \in C(X)$. Then

(i) $\mathcal{W}_{f} \subset M(X, S)$.

(ii) If $\mu \in M(X, S)$ and $h_{\mu}\left(\left.S\right|_{T}\right)+\int f d \mu=W(f)$, then $\mu \in W_{f}$.

(iii) Suppose the map $\mu \rightarrow h_{\mu}\left(\left.S\right|_{T}\right)$ is upper semicontinuous at all members of $W_{f}$. If $\mu$ is a signed measure on $X$, then $\mu \in \mathcal{W}_{f}$ iff $\mu \in M(X, S)$ and $h_{\mu}\left(\left.S\right|_{T}\right)+$ $\int f d \mu=W(f)$.

ProOF. (i) Let $\mu \in W_{f}$. To show that $\mu$ takes nonnegative values let $g \in C(X)$ with $g \geq 0$. We have

$$
\int g d \mu=-\int-g d \mu \geq-W(f-g)+W(f) \geq-[W(f)-\inf g]+W(f) \geq 0 .
$$

Therefore $\int g d \mu \geq 0$ and $\mu$ takes nonnegative values. We have $\mu(X)=1$, because for any integer $n$ (positive or negative) $\int n d \mu \leq W(f+n)-W(f)=n$. Finally, we have $\mu \in M(X, S)$, because for any integer $n$

$$
n \int(g \circ S-g) d \mu \leq W(f+n(g \circ S-g))-W(f)=0
$$

so that

$$
\int g \circ S d \mu=\int g d \mu \text {. }
$$

(ii) If $\mu \in M(X, S)$ and $h_{\mu}\left(\left.S\right|_{T}\right)+\int f d \mu=W(f)$, then

$$
W(f+g)-W(f) \geq h_{\mu}\left(\left.S\right|_{T}\right)+\int(f+g) d \mu-h_{\mu}\left(\left.S\right|_{T}\right)-\int f d \mu=\int g d \mu .
$$

Hence $\mu \in W_{f}$.

(iii) It remains to show, under the assumed condition, that if $\mu \in M(X, S) \cap \mathcal{W}_{f}$ then $h_{\mu}\left(\left.S\right|_{T}\right)+\int f d \mu=W(f)$. We have

$$
W(f+g)-\int(f+g) d \mu \geq W(f)-\int f d \mu \quad \forall g \in C(X),
$$

so by Theorem $2.1 h_{\mu}\left(\left.S\right|_{T}\right) \geq W(f)-\int f d \mu$. Therefore $h_{\mu}\left(\left.S\right|_{T}\right)+\int f d \mu \geq W(f)$, and the opposite inequality is clear.

We know from Lemma 2.2 that the assumptions of Theorem 2.2(iii) hold when $S$ is expansive.

It seems reasonable to call a measure that attains the supremum in

$$
\sup \left\{h_{\mu}\left(\left.S\right|_{T}\right)+\int f d \mu \mid \mu \in M(X, S)\right\}
$$

a relative equilibrium state for $f$. Theorem 2.2 implies that when the relative entropy map is upper semicontinuous the relative equilibrium states for $f$ are precisely the tangent functionals to $W$ at $f$.

We shall use the following corollary in the next section. 
COROLLARY 2.1. Suppose the relative entropy map, $\mu \rightarrow h_{\mu}\left(\left.S\right|_{T}\right)$, is upper semicontinuous on $M(X, S)$. If $\mu \in \mathcal{W}_{f}$ and $\mu=\int \nu d \tau(\nu)$ is the ergodic decomposition of $\mu$, then $\tau$-almost all $\nu$ belong to $W_{f}$.

Proof. By Theorem 2.2 we have $W(f)=h_{\mu}\left(\left.S\right|_{T}\right)+\int f d \mu$ and, using Lemma $3.2\left(\right.$ iii) of $[\mathbf{L}, \mathbf{W}]$, this gives $W(f)=\int\left[h_{\nu}\left(\left.S\right|_{T}\right)+\int f d \nu\right] d \tau(\nu)$. Since the integrand is majorised by $W(f)$, the result follows.

We would like to note the following fact.

THEOREM 2.3. Suppose that $S$ has finite topological entropy and $T$ has the property that every ergodic member of $M(Y, T)$ is an equilibrium state of some member of $C(Y)$. Then for each $f \in C(X)$

$$
W(f)=\sup \{P(S, f+\phi \circ \pi)-P(T, \phi) \mid \phi \in C(Y)\} .
$$

PROOF. From the finiteness of the topological entropy of $S$ we know that the pressure functions $P(S, \cdot), P(T, \cdot)$ are finite valued. Let

$$
Q(f)=\sup \{P(S, f+\phi \circ \pi)-P(T, \phi) \mid \phi \in C(Y)\} \text {. }
$$

Let $\varepsilon>0$. Choose $\phi_{\varepsilon} \in C(Y)$ with $Q(f)<P\left(S, f+\phi_{\varepsilon} \circ \pi\right)-P\left(T, \phi_{\varepsilon}\right)+\varepsilon$ and then, using the relative variational principle, choose $m_{\varepsilon} \in M(Y, T)$ with

$$
P\left(S, f+\phi_{\varepsilon} \circ \pi\right)<h_{m_{\varepsilon}}(T)+\int \phi_{\varepsilon} d m_{\varepsilon}+\int P(S, \pi, f) d m_{\varepsilon}+\varepsilon .
$$

Then $Q(f)<\int P(S, \pi, f) d m_{\varepsilon}+2 \varepsilon$, so that $Q(f) \leq W(f)$. To prove the converse, we use the assumption about equilibrium states of $T$. By the relative variational principle

$$
\begin{aligned}
Q(f) & =\sup _{\phi \in C(Y)}\left[\sup _{m \in M(Y, T)}\left(h_{m}(T)+\int \phi d m+\int P(S, \pi, f) d m\right)-P(T, \phi)\right] \\
& \geq \sup \left\{\int P(S, \pi, f) d m \mid m \text { is ergodic and } m \in M(Y, T)\right\} \\
& =W(f) . \quad \square
\end{aligned}
$$

It is known that when $T$ has finite topological entropy and its entropy map, $m \rightarrow h_{m}(T)$, is upper semicontinous on $M(Y, T)$ then every ergodic member of $M(Y, T)$ is an equilibrium state of some member of $C(Y)[\mathbf{R}$, p. 116]. (See also Corollary 3.2(ii) of this paper for an outline of the proof.) This holds when $T$ is expansive (in particular, when $T$ is a subshift).

One can also study other relative pressure functions. For each $m \in M(Y, T)$ we can define $W_{m}: C(X) \rightarrow R$ by $W_{m}(f)=\int P(S, \pi, f) d m$. By the relative variational principle we have

$$
W_{m}(f)=\sup \left\{h_{\mu}\left(\left.S\right|_{T}\right)+\int f d \mu \mid \mu \in M(X, S) \text { and } \mu \circ \pi^{-1}=m\right\} \text {. }
$$

We have the following results corresponding to Theorems 2.1 and 2.2.

THEOREM 2.4. Let $m \in M(Y, T)$ and let $M_{m}(X, S)=\left\{\mu \in M(X, S) \mid \mu \circ \pi^{-1}=\right.$ $m\}$. Let $\mu_{0} \in M_{m}(X, S)$. If the relative entropy map $\mu \rightarrow h_{\mu}\left(\left.S\right|_{T}\right)$ restricted to $M_{m}(X, S)$ is upper semicontinuous at $\mu_{0}$, then

$$
h_{\mu_{0}}\left(\left.S\right|_{T}\right)=\inf \left\{W_{m}(g)-\int g d \mu_{0} \mid g \in C(X)\right\} .
$$


The proof follows the same lines as that of Theorem 2.1. In the second part of the proof the set $C$ is replaced by

$$
C_{m}=\left\{(\mu, t) \in M_{m}(X, S) \times R \mid 0 \leq t \leq h_{\mu}\left(\left.S\right|_{T}\right)\right\} .
$$

THEOREM 2.5. Let $m \in M(Y, T)$ and $f \in C(X)$.

(i) All tangent functionals to $W_{m}$ at $f$ are in $M_{m}(X, S)$.

(ii) If $\mu \in M_{m}(X, S)$ and $h_{\mu}\left(\left.S\right|_{T}\right)+\int f d \mu=W_{m}(f)$, then $\mu$ is a tangent functional to $W_{m}$ at $f$.

(iii) Suppose the map $\mu \rightarrow h_{\mu}\left(\left.S\right|_{T}\right)$, restricted to $M_{m}(X, S)$, is upper semicontinuous at all tangent functionals to $W_{m}$ at $f$. If $\mu$ is a signed measure on $X$, then $\mu$ is a tangent functional to $W_{m}$ at $f$ iff $\mu \in M_{m}(X, S)$ and $h_{\mu}\left(\left.S\right|_{T}\right)+\int f d \mu=W_{m}(f)$.

The proof has only slight changes from that of Theorem 2.2. In (i) we have to show that a tangent functional $\mu$ to $W_{m}$ at $f$ satisfies $\mu \circ \pi^{-1}=m$. Let $\phi \in C(Y)$. Then

$$
\begin{aligned}
\int \phi \circ \pi d \mu & \leq W_{m}(f+\phi \circ \pi)-W_{m}(f) \\
& =\int[P(S, \pi, f+\phi \circ \pi)-P(S, \pi, f)] d m \\
& =\int \phi d m \quad \text { by the ergodic theorem. }
\end{aligned}
$$

Therefore $\int \phi d \mu \circ \pi^{-1} \leq \int \phi d m \forall \phi \in C(Y)$, and therefore $\int \phi d \mu \circ \pi^{-1}=\int \phi d m \forall \phi$ $\in C(Y)$. Hence $\mu \circ \pi^{-1}=m$.

The rest of the proof of Theorem 2.5 is like that of Theorem 2.2. Note that if $S$ is expansive, then the conditions of (iii) hold, so that $\forall m \in M(Y, T)$ and $\forall f \in C(X)$ the tangent functionals to $W_{m}$ at $f$ are exactly those members of $M_{m}(X, S)$ with $h_{\mu}\left(\left.S\right|_{T}\right)+\int f d \mu=W_{m}(f)$. We define relative equilibrium states of $f$ over $m$ to be those measures that project to $m$ and attain the maximum in $\sup \left\{h_{\nu}\left(\left.S\right|_{T}\right)+\int f d \nu \mid \nu \in M_{m}(X, S)\right\}$.

We also note that $W_{m}$ can be used to characterise $M_{m}(X, S)$. Let $m \in M(Y, T)$ and let $\mu$ be a finite signed measure on $(X, B(X))$. Then $\mu \in M_{m}(X, S)$ if and only if $\int f d \mu \leq W_{m}(f) \forall f \in C(X)$. This is a relativised version of a result of Ruelle.

One can also show that if $\mu$ is a finite signed measure on $(X, B(X))$ then $\mu \in$ $M(X, S)$ if and only if $\int f d \mu \leq W(f) \forall f \in C(X)$.

3. Compensation functions. In this section we shall consider the situation where $S: X \rightarrow X$ and $T: Y \rightarrow Y$ are both subshifts and $\pi: X \rightarrow Y$ is a continuous surjection with $\pi \circ S=T \circ \pi$. To say that $S: X \rightarrow X$ is a subshift means that $X$ is a shift-invariant closed subset of $\Omega_{k}=\{1,2, \ldots, k\}^{Z}$ for some $k \geq 1$ and $S$ is the restriction of the shift to $X$. A point of $X$ is written $x=\left(x_{n}\right)$, and $S x=x^{\prime}$, where $x_{n}^{\prime}=x_{n+1} \forall n \in Z$. The set $\{1,2, \ldots, k\}$ is called the symbol set for $X$. Similarly, $Y$ is a closed shift-invariant closed subset of $\Omega_{l}=\{1,2, \ldots, l\}^{Z}$ for some $l \geq 1$, and $T$ is the restriction of the shift to $Y$. A continuous surjection $\pi: X \rightarrow Y$ with $\pi S=T \pi$ has the following form: there are integers $p \leq q$ and a map $\theta:\{1,2, \ldots, k\}^{q-p+1} \rightarrow\{1,2, \ldots, l\}$ such that the $j$ th coordinate of $\pi(x)$ is $\theta\left(x_{p+j}, \ldots, x_{q+j}\right) \forall j \in Z, \forall x \in X[\mathbf{H}]$. Such maps $\pi$ are often called codes. Although we consider two-sided subshifts, the results of this section also hold for one-sided subshifts. 
By a block in $X$ we mean a finite string of symbols $\left(a_{0}, \ldots, a_{t}\right)$ such that there is some $x \in X$ with $x_{n}=a_{n}, 0 \leq n \leq t$. If $p \leq q$ are integers and $a_{n} \in$ $\{1,2, \ldots, k\}, 1 \leq n \leq q-p+1$, then ${ }_{p}\left[a_{1}, \ldots, a_{q-p+1}\right]_{q}$ denotes the set $\{x \in$ $\left.X \mid x_{n}=a_{n+1-p}, p \leq n \leq q\right\}$. Such a set is called a cylinder set.

If $\pi$ is such that the zeroth coordinate of $\pi(x)$ depends only on $x_{0}$, we say $\pi$ is a 1-block map. For any code $\pi$ we can always construct another subshift $X^{\prime}$ and a homeomorphism $H: X \rightarrow X^{\prime}$ such that $\pi \circ H^{-1}$ is a 1-block map. To do this (using the notation $\theta, p, q$ above), we take the symbol space for $X^{\prime}$ to be the set $\{1,2, \ldots, k\}^{q-p+1}$ and define $H$ by letting the $j$ th coordinate of $H(x)$ be $\left(x_{p+j}, x_{p+j+1}, \ldots, x_{q+j}\right) \in\{1,2, \ldots, k\}^{q-p+1}$. The set $X^{\prime}$ is then defined to be the image of $H$.

Our aim in this section is to show there exists $F \in C(X)$ with $P(S, F+\phi \circ \pi)$ $=P(T, \phi) \forall \phi \in C(Y)$, when $S, T$ are subshifts. We call such a function $F$ a compensation function. This result is motivated by the work of Boyle and Tuncel, who showed that such functions are related to how members of $M(Y, T)$ lift, by $\pi$, to members of $M(X, S)[\mathbf{B}, \mathbf{T}$, Theorem 3.6]. To show the existence of $F$, we shall use the function $W: C(X) \rightarrow R$ introduced in $\S 2$. We need the following result of Marcus, Petersen and Williams.

LEMMA $3.1[\mathbf{M}, \mathbf{P}, \mathbf{W}]$. If $S: X \rightarrow X, T: Y \rightarrow Y$ are subshifts and $\pi: X \rightarrow Y$ is a continuous surjection with $\pi \circ S=T \circ \pi$, then there is a closed subset $X_{1}$ of $X$ such that $S X_{1}=X_{1}$ and $\left.\pi\right|_{X_{1}}$ is bounded-to-one and maps $X_{1}$ onto $Y$.

PrOOF. By the above discussion we can assume $\pi$ is a one-block map. For each symbol $c$ of $Y$ fix a linear order on the set $\pi^{-1}(c)$ of symbols of $X$. These orderings give a natural lexicographical order on the set $\pi^{-1}(w)$ for each block $w$ of $Y$. For an $n$-block $w=\left(w_{1}, \ldots, w_{n}\right)$ in $Y$ and two symbols $a, b$ of $X$ with $\pi(a)=w_{1}, \pi(b)=w_{n}$, let $U(w ; a, b)$ denote the $n$-block $\left(a, x_{2}, x_{3}, \ldots, x_{n-1}, b\right)$ in $\pi^{-1}(w)$ which is smallest in the order on $\pi^{-1}(w)$, if such a block exists; otherwise put $U(w ; a, b)=\phi$. Let $X_{1}$ be the subshift determined by the collection of all blocks of the form $U(w ; a, b)$; i.e., $x=\left(x_{n}\right)_{-\infty}^{\infty} \in X_{1}$ if whenever $j \leq n$ the $(n-j+1)$-block $\left(x_{j}, x_{j+1}, \ldots, x_{n}\right)$ is a set of the form $U(w ; a, b)$. It is clear that $X \backslash X_{1}$ is open, and that $S X_{1}=X_{1}$.

To see that $\pi\left(X_{1}\right)=Y$, let $y=\left(y_{n}\right)$ be any point of $Y$. For each $n \geq 1$ choose any point $x^{(n)} \in X$ such that $\left(x_{-n}^{(n)}, x_{-(n-1)}^{(n)}, \ldots, x_{n}^{(n)}\right)$ is smallest in the order on $\pi^{-1}\left(y_{-n}, \ldots, y_{n}\right)$. Choose a convergent subsequence $\left\{x^{\left(n_{i}\right)}\right\}$ in $X$ and denote the limit by $x$. By construction of $x^{(n)}$ we have $\pi\left(x^{(n)}\right) \rightarrow y$, and so $\pi(x)=y$. We have $x \in X_{1}$, because if $j \leq n$, then $\left(x_{j}, \ldots, x_{n}\right)$ is a subblock of $\left(x_{-N}^{(N)}, \ldots, x_{N}^{(N)}\right)$ for some large $N$, and hence $\left(x_{j}, \ldots, x_{n}\right)=U\left(\left(y_{j}, \ldots, y_{n}\right) ; x_{j}, x_{n}\right)$.

We now show that $\left.\pi\right|_{X_{1}}$ is bounded-to-one. Let $y \in Y$. For each $n \geq 1$, $\pi^{-1}\left(y_{-n}, \ldots, y_{n}\right) \cap X_{1}$ is a union of at most $k^{2}$ blocks in $X$ of length $2 n+1$ (where $k$ is the number of symbols in $X)$. Hence $\pi^{-1}(y)$ contains at most $k^{2}$ points of $X$.

COROLlaRY 3.1. Let $X_{1}$ be the set obtained in Lemma 3.1. For every $\phi \in$ $C(Y)$ we have $P\left(\left.S\right|_{X_{1}},\left.\phi \circ \pi\right|_{X_{1}}\right)=P(T, \phi)$. 
PROOF. Since $\pi_{1}=\pi \mid X_{1}$ is bounded-to-one we have, $h_{\mu}\left(\left.S\right|_{X_{1}}\right)=h_{\mu \circ \pi_{1}^{-1}}(T)$ for every $\mu \in M\left(X_{1}, S \mid X_{1}\right)[\mathbf{P}, \mathbf{T}$, p. 56]. The result follows from the variational principle.

From now on suppose that $X_{1}$ is a chosen subset of $X$ with the properties listed in Lemma 3.1. All the remaining results in this section hold when $S: X \rightarrow X, T: X \rightarrow$ $Y$ are homeomorphisms of compact metric spaces with finite topological entropy and with upper semicontinuous entropy maps, provided we assume there is a closed subset $X_{1}$ of $X$ with $S X_{1}=X_{1}, \pi X_{1}=Y$ and $\left.\pi\right|_{X_{1}}$ a bounded-to-one map.

We now bring in the function $W: C(X) \rightarrow R$ studied in $\S 2$.

LEMMA 3.2. For $f \in C(X)$ satisfying $f \equiv 0$ on $X_{1}$, the following conditions are mutually equivalent.

(i) $f$ is a compensation function.

(ii) $W(f)=0$.

(iii) $W(f) \leq 0$.

Proof. The implication (i) $\Rightarrow$ (ii) follows from Theorem 2.3. Since the implication (ii) $\Rightarrow$ (iii) is clear, we only need to show (iii) implies (i). Assume $W(f) \leq 0$. From Theorem 2.3 we have $P(S, f+\phi \circ \pi) \leq P(T, \phi) \forall \phi \in C(Y)$. However

$$
P(S, f+\phi \circ \pi) \geq P\left(\left.S\right|_{X_{1}},\left.(f+\phi \circ \pi)\right|_{X_{1}}\right)=P\left(\left.S\right|_{X_{1}},\left.\phi \circ \pi\right|_{X_{1}}\right)=P(T, \phi),
$$

so that $P(S, f+\phi \circ \pi)=P(T, \phi) \forall \phi \in C(Y)$.

We shall find a function $f \in C(X)$ with $f \equiv 0$ on $X_{1}$ and $W(f) \leq 0$ by using the following special cases of a theorem of Israel. (Markley and Paul have used a version of Israel's theorem to deal with a related problem $[\mathbf{M}, \mathbf{P}]$.)

LEMMA 3.3 [I, p. 115]. Let $X$ be a compact metric space, and let $W: C(X)$ $\rightarrow R$ be convex and continuous. Suppose $\mathcal{N}$ is a closed cone in $C(X)$ with apex 0. Let $f_{0} \in C(X)$ and let $\mu_{0} \in M(X)$ be $W$-bounded (i.e., $\exists c \in R$ such that $\left.\int g d \mu_{0} \leq c+W(g) \forall g \in C(X)\right)$. For each $\varepsilon>0$ there exists $f \in C(X)$ and $\mu \in C(X)^{*}$ such that

(i) $f \in f_{0}+\mathcal{N}$,

(ii) $\mu$ is a tangent functional to $W$ at $f$,

(iii) $\int h d \mu \geq \int h d \mu_{0}-\varepsilon\|h\| \forall h \in \mathcal{N}$.

We shall use this result with $\mathcal{N}=\left\{h \in C(X) \mid h \equiv 0\right.$ on $X_{1}$ and $\left.h \leq 0\right\}$. Note that any $\mu_{0} \in M(X, S)$ is $W$-bounded since

$$
W(g) \geq h_{\mu_{0}}\left(\left.S\right|_{T}\right)+\int g d \mu_{0} \geq \int g d \mu_{0} .
$$

We can now prove the main result of this section.

THEOREM 3.1. Let $S: X \rightarrow X, T: Y \rightarrow Y$ be subshifts and let $\pi: Y \rightarrow Y$ be a continuous surjection with $\pi \circ S=T \circ \pi$. There exists $F \in C(X)($ actually $F \in \mathcal{N})$ which is a compensation function.

Proof. By Lemma 3.2 it is enough to find some $f \in \mathcal{N}$ with $W(f) \leq 0$. Apply Lemma 3.3 with $f_{0} \equiv 0, \mu_{0} \in M\left(X_{1},\left.S\right|_{X_{1}}\right)$ and $\varepsilon<1$. There exist $f \in \mathcal{N}$ and $\mu \in \mathcal{W}_{f}$ such that $\int h d \mu \geq \int h d \mu_{0}-\varepsilon\|h\| \forall h \in \mathcal{N}$.

We first show that $\mu\left(X_{1}\right)>0$. For each $n \geq 1$ let $U_{n}=\left\{x \in X \mid d\left(x, X_{1}\right)<1 / n\right\}$, where $d$ is a metric on $X$. Choose $h_{n} \in C(X)$ such that $h_{n} \equiv 0$ on $X_{1}, h_{n} \equiv-1$ 
on $X \backslash U_{n}$, and $h_{n}(x) \in[-1,0] \forall x \in X$. Then $h_{n} \in \mathcal{N}$ and $-\mu\left(X \backslash U_{n}\right) \geq \int h_{n} d \mu \geq$ $\int h_{n} d \mu_{0}-\varepsilon=-\varepsilon$. Therefore $\mu\left(X \backslash U_{n}\right) \leq \varepsilon \forall n \geq 1$, so that $\mu\left(X_{1}\right) \geq 1-\varepsilon$.

By Corollary 2.1 we can choose some $\mu_{1}$ in the ergodic decomposition of $\mu$ so that $\mu_{1} \in \mathcal{W}_{f}$ and $\mu_{1}\left(X_{1}\right)>0$. Since $\mu_{1}$ is ergodic, $\mu_{1}\left(X_{1}\right)=1$. Since $\mu_{1} \in \mathcal{W}_{f}$, we have, by Theorem 2.2 ,

$$
\begin{aligned}
W(f) & =h_{\mu_{1}}(S)-h_{\mu_{1} \circ \pi^{-1}}(T)+\int f d \mu_{1} \\
& =h_{\mu_{1}}\left(\left.S\right|_{X_{1}}\right)-h_{\mu_{1} \circ \pi^{-1}}(T)+\int_{X_{1}} f d \mu_{1} \\
& =0 \text { since }\left.\pi\right|_{X_{1}} \text { is bounded to one and } f \equiv 0 \text { on } X_{1} .
\end{aligned}
$$

Recall from Theorem 2.2 that the tangent functionals to $W$ at $f$ are the relative equilibrium states of $f$.

THEOREM 3.2. We can choose a compensation function $F_{1} \in C(X)$ with the additional properties:

(i) $W_{F_{1}}=M\left(X_{1},\left.S\right|_{X_{1}}\right)$,

(ii) $\forall \phi \in C(Y)$ the equilibrium states of $F_{1}+\phi \circ \pi$ with respect to $S$ are exactly the equilibrium states of $\left.\phi \circ \pi\right|_{X_{1}}$ with respect to $\left.S\right|_{X_{1}}$.

PROOF. We have constructed a compensation function $F \in \mathcal{N}$. Choose $F_{1} \in \mathcal{N}$ so that $F_{1}(x)<F(x) \forall x \notin X_{1}$. Then $W\left(F_{1}\right) \leq W(F)=0$, so, by Lemma $3.2, F_{1}$ is a compensation function and $W\left(F_{1}\right)=0$.

(i) If $\mu \in \mathcal{W}_{F_{1}}$ and $\mu\left(X_{1}\right) \neq 1$, then

$$
0=W\left(F_{1}\right)=h_{\mu}\left(\left.S\right|_{T}\right)+\int F_{1} d \mu<h_{\mu}\left(\left.S\right|_{T}\right)+\int F d \mu \leq W(F)=0 .
$$

Therefore $\mu\left(X_{1}\right)=1$. Hence $\mathcal{W}_{F_{1}} \subset M\left(X_{1},\left.S\right|_{X_{1}}\right)$. The opposite inequality is clear.

(ii) If $\mu$ is an equilibrium state for $F_{1}+\phi \circ \pi$ and $\mu\left(X_{1}\right) \neq 1$, then

$$
\begin{aligned}
P(T, \phi) & =P\left(S, F_{1}+\phi \circ \pi\right)=h_{\mu}(S)+\int F_{1} d \mu+\int \phi \circ \pi d \mu \\
& <h_{\mu}(S)+\int F d \mu+\int \phi \circ \pi d \mu \leq P(S, F+\phi \circ \pi)=P(T, \phi) .
\end{aligned}
$$

Therefore $\mu\left(X_{1}\right)=1$, and $\mu$ is an equilibrium state for $\phi \circ \pi$ with respect to $\left.S\right|_{X_{1}}$. The converse is clear.

We now consider some equivalent conditions for a function $F \in C(X)$ to be a compensation function. Although we state the results for subshifts $S, T$, the results are valid when $S$ and $T$ each have finite topological entropy and upper semicontinuous entropy map. We shall use the following generalisation of a result of Israel.

LEMMA 3.4. Suppose $F \in C(X)$ is given. If $m \in M(Y, T)$ is ergodic there is some $\mu \in M(X, S)$ with $\mu \circ \pi^{-1}=m$ and $\mu$ is an equilibrium state of $F+\phi \circ \pi$ for some $\phi \in C(Y)$.

PROOF. Let $\mu_{0}$ be any element of $M(X, S)$ with $\mu_{0} \circ \pi^{-1}=m$. By applying Lemma 3.3 to the pressure $P(S, \cdot): C(X) \rightarrow R$, with $\mathcal{N}=\{\phi \circ \pi \mid \phi \in C(Y)\}, f_{0}=$ $F$, and $\varepsilon=\frac{1}{2}$, there is $\mu \in M(X, S)$ which is an equilibrium state of $F+\phi \circ \pi$ 
for some $\phi \in C(Y)$ and $\left\|\mu \circ \pi^{-1}-m\right\|<\frac{1}{2}$. Let $\mu=\int \nu d \tau(\nu)$ be the ergodic decomposition of $\mu$. Then $\mu \circ \pi^{-1}=\int p d\left(\tau \circ \tilde{\pi}^{-1}\right)(p)$ is the ergodic decomposition $\mu \circ \pi^{-1}$, where $\tilde{\pi}: M(X, S) \rightarrow M(Y, T)$ is the map induced by $\pi$. But $\left\|\mu \circ \pi^{-1}-m\right\|=$ $\left\|\tau \circ \tilde{\pi}^{-1}-\delta_{m}\right\|<\frac{1}{2}$ (see p. 167 of $\left.[\mathbf{R}]\right)$, so that $\left(\tau \circ \tilde{\pi}^{-1}\right)(m)>0$. Since $\tau\left(\tilde{\pi}^{-1} m\right)>0$ and the set of equilibrium states of $F+\phi \circ \pi$ is a face of $M(X, S)[\mathbf{R}$, p. 51], we conclude that some lift of $m$ is an equilibrium state of $F+\phi \circ \pi$.

COROLLARY 3.2 [I, p. 117]. (i) If $m \in M(Y, T)$ is ergodic, there exists some $\mu \in M(X, S)$ which is an equilibrium state of some function $\phi \circ \pi, \phi \in C(Y)$, and $\mu \circ \pi^{-1}=m$.

(ii) Every ergodic $m \in M(Y, T)$ is the equilibrium state of some $\phi \in C(Y)$.

ProOF. (i) Put $F=0$ and apply Lemma 3.4.

(ii) Put $X=Y, S=T$ and $\pi=$ id. This is the result of Israel we used in the proof of Lemma 3.2.

THEOREM 3.3. Let $S: X \rightarrow X, T: Y \rightarrow Y$ be subshifts, and let $\pi: X \rightarrow Y$ be a continuous surjection with $\pi \circ S=T \circ \pi$. For $F \in C(X)$ the following conditions are equivalent:

(i) $F$ is a compensation function.

(ii) $\int P(S, \pi, F) d m=0 \forall m \in M(Y, T)$.

(iii) $\{y \in Y \mid P(S, \pi, F)(y)=0\}$ is a total probability set for $T$ (i.e., has measure one for each $m \in M(Y, T))$.

Proof. Assume (i) holds. We shall show $\int P(S, \pi, F) d m=0$ for every ergodic $m \in M(Y, T)$. Since each ergodic $m$ is the equilibrium state of some $\psi \in C(Y)$ and we know

$$
\begin{gathered}
\sup \left\{h_{p}(T)+\int P(S, \pi, F) d p+\int \psi d p \mid p \in M(Y, T)\right\} \\
=\sup \left\{h_{p}(T)+\int \psi d p \mid p \in M(Y, T)\right\}
\end{gathered}
$$

we must have $\int P(S, \psi, F) d m \leq 0$ for all ergodic $m \in M(Y, T)$ and hence for all $m \in M(Y, T)$. By Lemma 3.4 we know that for each ergodic $m \in M(Y, T)$ there is some $\mu \in M(X, S)$, which is the equilibrium state of $F+\phi \circ \pi$ for some $\phi \in C(Y)$, such that $\mu \circ \pi^{-1}=m$. Then we have

$$
\begin{aligned}
P(S, F+\phi \circ \pi) & =h_{m}(T)+\int \phi d m+\int P(S, \pi, F) d m \\
& \leq h_{m}(T)+\int \phi d m \leq P(T, \phi),
\end{aligned}
$$

and since $P(S, F+\phi \circ \pi)=P(T, \phi)$, we conclude that $\int P(S, \pi, F) d m=0$. Therefore (ii) holds.

If we assume (ii), then since $P(S, \pi, F)$ is $T$-invariant we have $P(S, \pi, F)=0$ a.e. for every ergodic member of $M(Y, T)$ and hence for every member of $M(Y, T)$. Hence (iii) holds. 
Now suppose (iii) is true. Then, if $\phi \in C(Y)$,

$$
\begin{aligned}
P(S, F+\phi \circ \pi) & =\sup \left\{h_{m}(T)+\int P(S, \pi, F) d m+\int \phi d m \mid m \in M(Y, T)\right\} \\
& =\sup \left\{h_{m}(T)+\int \phi d m \mid m \in M(Y, T)\right\}=P(T, \phi) .
\end{aligned}
$$

One relationship between compensation functions and equilibrium states is the following result of Boyle and Tuncel.

COROLlaRY $3.3[\mathbf{B}, \mathbf{T}]$. Suppose $F \in C(X)$ is a compensation function. Let $\phi \in C(Y)$. If $\mu$ is an equilibrium state for $F+\phi \circ \pi$, then $\mu \circ \pi^{-1}$ is an equilibrium state for $\phi$.

PROOF.

$$
\begin{aligned}
P(T, \phi) & =P(S, F+\phi \circ \pi)=h_{\mu}(S)+\int F d \mu+\int \phi \circ \pi d \mu \\
& =\sup \left\{h_{\mu_{1}}(S)+\int F d \mu_{1}+\int \phi \circ \pi d \mu_{1} \mid \mu_{1} \circ \pi^{-1}=\mu \circ \pi^{-1}\right\} \\
& =h_{\mu \circ \pi^{-1}}(T)+\int P(S, \pi, F) d \mu \circ \pi^{-1}+\int \phi d \mu \circ \pi^{-1}
\end{aligned}
$$

by the relative variational principle

$$
=h_{\mu \circ \pi^{-1}}(T)+\int \phi d \mu \circ \pi^{-1} \text { by (ii) of Theorem 3.3. }
$$

We can sharpen the above result to obtain

CoROLlaRY 3.4. Suppose $F \in C(X)$ is a compensation function. Let $\mu \in$ $M(X, S)$ and $\phi \in C(Y)$. Then $\mu$ is an equilibrium state of $F+\phi \circ \pi$ iff $\mu \circ \pi^{-1}$ is an equilibrium state of $\phi$ and $\mu$ is a relative equilibrium state of $F$ over $\mu \circ \pi^{-1}$.

PRoOF. If $\mu$ is an equilibrium state of $F+\phi \circ \pi$, then $\mu \circ \pi^{-1}$ is an equilibrium state of $\phi$ by Corollary 3.3. Also,

$$
h_{\mu}\left(\left.S\right|_{T}\right)+\int F d \mu+h_{\mu \circ \pi^{-1}}(T)+\int \phi d \mu \circ \pi^{-1}=P(S, F+\phi \circ \pi),
$$

so, by the relative variational principle, $\mu$ is a relative equilibrium state of $F$ over $\mu \circ \pi^{-1}$.

Conversely, if $\mu$ is a relative equilibrium state of $F$ over $\mu \circ \pi^{-1}$ and $\mu \circ \pi^{-1}$ is an equilibrium state of $\phi$, then

$$
\begin{aligned}
h_{\mu}(S)+\int F d \mu+ & \int \phi \circ \pi d \mu \\
& =h_{\mu}\left(\left.S\right|_{T}\right)+\int F d \mu+h_{\mu \circ \pi^{-1}}(T)+\int \phi d \mu \circ \pi^{-1} \\
& =\int P(S, \pi, F) d \mu \circ \pi^{-1}+h_{\mu \circ \pi^{-1}}(T)+\int \phi d \mu \circ \pi^{-1} \\
& =h_{\mu \circ \pi^{-1}}(T)+\int \phi d \mu \circ \pi^{-1}=P(T, \phi)=P(S, F+\phi \circ \pi) .
\end{aligned}
$$


Therefore $\mu$ is an equilibrium state of $F+\phi \circ \pi$.

When we have a compensation function in $C(Y)$, there is a fourth equivalent condition:

TheOREM 3.4. Let $S: X \rightarrow X, T: Y \rightarrow Y$ be subshifts, and let $\pi: X \rightarrow Y$ be a continuous surjection with $\pi \circ S=T \circ \pi$. For $G \in C(Y)$ the three equivalent statements in Theorem 3.3 (with $F$ replaced by $G \circ \pi$ ) are also equivalent to

(iv) $\int P(S, \pi, 0) d m=-\int G d m \forall m \in M(Y, T)$.

PrOOF. If (iv) holds, then for $\phi \in C(Y)$ the relative variational principle gives

$$
\begin{aligned}
P(S, G \circ \pi+\phi \circ \pi) & \\
& =\sup \left\{h_{m}(T)+\int P(S, \pi, 0) d m+\int(G+\phi) d m \mid m \in M(Y, T)\right\} \\
& =\sup \left\{h_{m}(T)+\int \phi d m \mid m \in M(Y, T)\right\}=P(T, \phi) .
\end{aligned}
$$

Therefore $G \circ \pi$ is a compensation function.

Now suppose $G \circ \pi$ is a compensation function. For every ergodic member of $M(Y, T)$ we have

$$
P(S, \pi, G \circ \pi)(y)=P(S, \pi, 0)(y)+\lim _{n \rightarrow \infty} \frac{1}{n} \sum_{i=0}^{n-1} G\left(T^{i} y\right) \quad \text { a.e. }
$$

By (ii) of Theorem 3.3 we have $0=\int P(S, \pi, 0) d m+\int G d m$ for every ergodic $m$ and hence for all $m \in M(Y, T)$.

When we know $G \circ \pi$, for $G \in C(Y)$, is a compensation function then Corollary 3.3 asserts that, for any $\psi \in C(Y)$ and any equilibrium state $\mu$ for $\psi \circ \pi, \mu \circ \pi^{-1}$ is an equilibrium state for $\psi-G$. (Put $\phi=\psi-G$ in Corollary 3.3.) Corollary 3.4, in the case when $G \circ \pi$ is a compensation function for some $G \in C(Y)$, says the following. Let $\psi \in C(Y)$ and $\mu \in M(X, S)$. The measure $\mu$ is an equilibrium state of $\psi \circ \pi$ iff $\mu \circ \pi^{-1}$ is an equilibrium state of $\psi-G$ and $\mu$ is a measure of maximal relative entropy over $\mu \circ \pi^{-1}$. The last statement means

$$
h_{\mu}\left(\left.S\right|_{T}\right)=\sup \left\{h_{\nu}\left(\left.S\right|_{T}\right) \mid \nu \in M(X, S) \text { and } \nu \circ \pi^{-1}=\mu \circ \pi^{-1}\right\} .
$$

4. Compensation functions and equilibrium states for subshifts of finite type. In this section both $S: X \rightarrow X, T: Y \rightarrow Y$ will be topologically mixing subshifts of finite type and $\pi: X \rightarrow Y$ will be a continuous surjection with $\pi \circ S=T \circ \pi$. We shall relate compensation functions to equilibrium states and discuss different types of quotient maps $\pi$. We get a new way of viewing some of the results of $[\mathbf{B}, \mathbf{T}]$.

For convenience we shall assume our subshifts of finite type are of order one. This means that if $\{1,2, \ldots, k\}$ are the symbols for $X$ then there is a $k \times k$ matrix $A=\left[a_{i j}\right]$, with $a_{i j} \in\{0,1\}$ for each $i, j$, such that $X$ consists of all bisequences $\left(x_{n}\right)_{-\infty}^{\infty}$ with $a_{x_{n} x_{n+1}}=1 \forall n \in Z$. Similarly, $Y$ is defined by a $0-1$ matrix $B$. If $X$ or $Y$ is a subshift of finite type of higher order, the results of this section remain valid; only the condition (*) below needs restating slightly. Recall that $S: X \rightarrow X$ is topologically mixing iff there is some integer $p>0$ with $A^{p}$ having all of its entries strictly positive. 
For a topologically mixing subshift of finite type, $S: X \rightarrow X$, there is a large subset of $C(X)$ consisting of functions with unique equilibrium states. We now summarise what we need from this theory. For $x=\left(x_{n}\right)_{-\infty}^{\infty} \in X$ we let $x^{+}$denote the one-sided sequence $x^{+}=\left(x_{0}, x_{1}, x_{2}, \ldots\right)$. Let $X^{+}$denote the collection of all these points. If $\left(a_{-n}, \ldots, a_{-1}\right)$ is a block of symbols so that $\left(a_{-n}, \ldots, a_{-1}, x_{0}\right)$ is allowed in $X$, then $\left(a_{-n}, \ldots, a_{-1}, x^{+}\right)$denotes the one-sided point $\left(a_{-n}, a_{-(n-1)}, \ldots, a_{-1}\right.$, $\left.x_{0}, x_{1}, x_{2}, \ldots\right)$. A function $f \in C(X)$ is called a one-sided function if $f(x)=f\left(x^{\prime}\right)$ whenever $x_{n}=x_{n}^{\prime} \forall n \geq 0$. It then makes sense for $f$ to be evaluated on one-sided sequences $\left(x_{0}, x_{1}, \ldots\right)$. Two functions $f_{1}, f_{2} \in C(X)$ are said to be cohomologous if there exists $h \in C(X)$ with $f_{1}-f_{2}=h \circ S-h$.

We now define a class of functions $\mathcal{F}(X)$. We say $f \in \mathcal{F}(X)$ if $f \in C(X)$ and $f$ is cohomologous to a one-sided function $f_{0}$ which satisfies the following condition:

(*) $C_{f_{0}}\left(x^{+}, z^{+}\right)=\sup _{n \geq 1}\left[\sup \left\{\sum_{i=1}^{n}\left(f_{0}\left(a_{-i}, \ldots, a_{-1}, x^{+}\right)-f_{0}\left(a_{-i}, \ldots, a_{-1} z^{+}\right)\right) \mid\right.\right.$

$$
\left.\left.\left(a_{-n}, \ldots, a_{-1}, x_{0}\right) \text { is an allowable block in } X\right\}\right]
$$

exists when $x_{0}=z_{0}$, is bounded above by a constant $C_{f_{0}}$, and $C_{f_{0}}\left(x^{+}, z^{+}\right) \rightarrow 0$ as $d\left(x^{+}, z^{+}\right) \rightarrow 0$. (Here $d$ is a metric on $X^{+}$.)

Then $\mathcal{F}(X)$ is a linear subspace of $C(X)$ and is dense in $C(X)$ since it contains all functions depending on only finitely many coordinates of $X$. The space $\mathcal{F}(X)$ includes all $f$ with $\operatorname{var}_{n}(f) \leq C \alpha^{n}(0<\alpha<1)$, studied in $[\mathbf{B}, \mathbf{R}]$, and the one-sided functions with $\sum_{n=1}^{\infty} \operatorname{var}_{n}(f)<\infty$ studied in $\left[\mathbf{W}_{1}\right]$. We have $f \in \mathcal{F}(X)$ implies $f \circ S, f \circ S^{-1} \in \mathcal{F}(X)$.

Recall that for $\mu \in M(X, S)$ the information function $I_{\mu}: X \rightarrow R$ is defined, almost everywhere, by $I_{\mu}=I_{\mu}\left(A / S^{-1} A\right)$, where $A$ is the $\sigma$-algebra generated by the positive coordinates. In $\left[\mathbf{L}, \mathbf{W}_{1}, \mathbf{W}_{2}\right]$ the case when $I_{\mu}$ has a continuous version was studied. As we note below in Proposition 4.1, we shall be interested in measures $\mu$ for which $I_{\mu}$ has a continuous version, and then $I_{\mu}$ will denote the continuous version. A Markov measure for $S$ is a $\mu \in M(X, S)$ for which $I_{\mu}$ has a continuous version depending on only a fixed finite number of coordinates of $x \in X$. Markov measures are precisely those measures which are equilibrium states of functions depending on a finite number of coordinates (see Proposition 4.1(iii)). Under this definition the support of a Markov measure on $X$ is the whole of $X$. We have the following results: the proofs can be found in $\left[\mathbf{R}, \mathbf{B}, \mathbf{L}, \mathbf{W}_{\mathbf{1}}, \mathbf{W}_{\mathbf{2}}\right]$.

Proposition 4.1. Let $S: X \rightarrow X$ be a topologically mixing subshift of finite type. Let $f \in \mathcal{F}(X)$. The following statements hold:

(i) The function $f$ has a unique equilibrium state $\mu_{f}$.

(ii) The measure $\mu_{f}$ is the only $S$-invariant probability with the property that there are constants $\alpha, \beta>0$ satisfying

$$
\alpha \leq \mu_{f}\left(0\left[x_{0}, \ldots, x_{n-1}\right]_{n-1}\right) \exp \left(n P(S, f)-\left(S_{n} f\right)(x)\right) \leq \beta \quad \forall n \geq 1, \forall x \in X .
$$

(iii) The function $I_{\mu_{f}}$ has a continuous version which is a one-sided function satisfying condition $(*)$. There is a constant $c$ such that $f$ is cohomologous to $c-I_{\mu_{f}}$. Also $P\left(S,-I_{\mu_{f}}\right)=0$, so that $c=P(S, f)$. If $f$ depends on only finitely many coordinates, then so does $I_{\mu_{f}}$. 
(iv) If $f, g \in \mathcal{F}(X)$, then $\mu_{f}=\mu_{g}$ iff $\exists c \in R$ such that $f$ is cohomologous to $g+c$.

On the space $Y$ we have the family $\mathcal{F}(Y)$, and $\pi$ relates $\mathcal{F}(Y)$ and $\mathcal{F}(X)$ as follows.

LEMMA 4.1. If $\phi \in \mathcal{F}(Y)$, then $\phi \circ \pi \in \mathcal{F}(X)$.

PROOF. Choose $p \leq 0$ so that the zeroth coordinate of $\pi\left(S^{p} x\right)$ depends only on the coordinates $\left(x_{0}, x_{1}, x_{2}, \ldots\right)$. Then the zeroth coordinate of $\pi\left(S^{p} x\right)$ has the form $\theta\left(x_{0}, \ldots, x_{l-1}\right)$ for some $l$. It suffices to show that $\phi \in \mathcal{F}(Y)$ implies $\phi \circ \pi \circ S^{p} \in$ $\mathcal{F}(X)$. We know $\phi$ is cohomologous to a one-sided function $\phi_{0}$ with $C_{\phi_{0}}\left(y^{+}, u^{+}\right) \rightarrow 0$ as $d\left(y^{+}, u^{+}\right) \rightarrow 0$. Then $\phi \circ \pi \circ S^{p}$ is cohomologous to $\phi_{0} \circ \pi \circ S^{p}$, which is one-sided, and

$$
C_{\phi_{0} \circ \pi \circ S^{p}}\left(x^{+}, z^{+}\right) \leq C_{\phi_{0}}+2 l\left\|\phi_{0}\right\| \quad \text { if } x_{0}=z_{0}
$$

and

$$
C_{\phi_{0} \circ \pi \circ S^{p}}\left(x^{+}, z^{+}\right) \leq C_{\phi_{0}}\left(\pi S^{p} x^{+}, \pi S^{p} z^{+}\right) \quad \text { if }\left(x_{0}, \ldots, x_{l}\right)=\left(z_{0}, \ldots, z_{l}\right) .
$$

Therefore $\phi_{0} \circ \pi \circ S^{p}$ satisfies condition (*), and hence $\phi \circ \pi \circ S^{p} \in \mathcal{F}(X)$.

COROLlaRY 4.1. If $S: X \rightarrow X$ and $T: Y \rightarrow Y$ are topologically conjugate by a homeomorphism $\Phi: X \rightarrow Y$, then $f \in \mathcal{F}(X)$ iff $f \circ \Phi^{-1} \in \mathcal{F}(Y)$.

We use Greek letters such as $\phi$ for members of $C(Y)$, and if $\phi \in \mathcal{F}(Y)$ we denote its unique equilibrium state by $m_{\phi}$. We denote elements of $C(X)$ by $f, g, h$, etc.; and when $f \in \mathcal{F}(X)$, we denote its unique equilibrium state by $\mu_{f}$.

From Lemma 4.1 we know that if $f \in \mathcal{F}(X)$ and $\phi \in \mathcal{F}(Y)$ then $f+\phi \circ \pi \in \mathcal{F}(X)$.

When $F \in \mathcal{F}(X)$, we have the following equivalent conditions for $F+c$ to be a compensation function for some $c \in R$.

THEOREM 4.1. Let $S: X \rightarrow X, T: Y \rightarrow Y$ be topologically mixing subshifts of finite type, and let $\pi: X \rightarrow Y$ be a continuous surjection with $\pi \circ S=T \circ \pi$. The following statements are equivalent for $F \in \mathcal{F}(X)$ :

(i) $\exists c \in R$ such that $c+F$ is a compensation function.

(ii) for each $\phi \in C(Y)$ which has a unique equilibrium state $m_{\phi}$, we have $\mu \circ \pi^{-1}=$ $m_{\phi}$ for every equilibrium state $\mu$ of $F+\phi \circ \pi$.

(iii) $\forall \phi \in \mathcal{F}(Y)$ we have $\mu_{F+\phi \circ \pi} \circ \pi^{-1}=m_{\phi}$.

(iv) $\exists \phi \in \mathcal{F}(Y)$ with $\mu_{F+\phi \circ \pi} \circ \pi^{-1}=m_{\phi}$.

(v) $\forall \phi \in \mathcal{F}(Y) \mu_{F+\phi \circ \pi}$ is the unique relative equilibrium state for $F$ over $m_{\phi}$.

ProOF. We have (i) $\Rightarrow$ (ii) by Corollary 3.3. The implications (ii) $\Rightarrow$ (iii) and (iii) $\Rightarrow$ (iv) are clear. We now show (iv) $\Rightarrow\left(\right.$ i). Let $\phi \in \mathcal{F}(Y)$ satisfy $\mu_{F+\phi \circ \pi} \circ \pi^{-1}=$ $m_{\phi}$. In this proof $\left[y_{0}, \ldots, y_{n-1}\right]$ means $0\left[y_{0}, \ldots, y_{n-1}\right]_{n-1}$. By Proposition 4.1 $\forall \psi \in \mathcal{F}(Y)$ there are positive constants $c_{1}(\psi), c_{2}(\psi)$ with

$$
c_{1}(\psi) \leq m_{\psi}\left(\left[y_{0}, \ldots, y_{n-1}\right]\right) \exp \left(n P(T, \psi)-\left(T_{n} \psi\right)(y)\right) \leq c_{2}(\psi) \quad \forall n \geq 1 \forall y \in Y,
$$

where $\left(T_{n} \psi\right)(y)$ means $\sum_{i=0}^{n-1} \psi\left(T^{i} y\right)$. Also there are positive constants $a_{1}(\psi), a_{2}(\psi)$ so that

$$
\begin{aligned}
a_{1}(\psi) & \leq \mu_{F+\psi \circ \pi}\left(\left[x_{0}, \ldots, x_{n-1}\right]\right) \exp \left(n P(S, F+\psi \circ \pi)-\left(S_{n} F\right)(x)-\left(T_{n} \psi\right)(\pi x)\right) \\
& \leq a_{2}(\psi) \quad \forall n \geq 1 \forall x \in X
\end{aligned}
$$


Therefore

$$
\begin{aligned}
\frac{a_{1}(\psi)}{a_{2}(\phi)} \leq & \frac{\mu_{F+\psi \circ \pi}\left(\left[x_{0}, \ldots, x_{n-1}\right]\right)}{\mu_{F+\phi \circ \pi}\left(\left[x_{0}, \ldots, x_{n-1}\right]\right)} \\
& \times \exp \left(n(P(S, F+\psi \circ \pi)-P(S, F+\phi \circ \pi))+\left(T_{n} \phi\right)(\pi x)-\left(T_{n} \psi\right)(\pi x)\right) \\
\leq & \frac{a_{2}(\psi)}{a_{1}(\phi)}
\end{aligned}
$$

If the zeroth coordinate of $\pi(x)$ depends on the coordinates $\left(x_{q}, \ldots, x_{q+l}\right)$ of $x$, then $\pi^{-1}\left[y_{0}, \ldots, y_{n-1}\right]$ is a union of blocks of length $n+l+1$, and we have positive constants $b_{1}(\psi), b_{2}(\psi)$ such that

$$
\begin{aligned}
b_{1}(\psi) \leq & \frac{\mu_{F+\psi \circ \pi}\left(\pi^{-1}\left[y_{0} \cdots y_{n-1}\right]\right)}{\mu_{F+\phi \circ \pi}\left(\pi^{-1}\left[y_{0} \cdots y_{n-1}\right]\right)} \\
& \times \exp \left(n(P(S, F+\psi \circ \pi)-P(S, F+\phi \circ \pi))+\left(T_{n} \phi\right)(y)-\left(T_{n} \psi\right)(y)\right) \\
\leq & b_{2}(\psi) .
\end{aligned}
$$

Using $\mu_{F+\phi \circ \pi} \circ \pi^{-1}=m_{\phi}$, we have

$$
\begin{aligned}
\frac{b_{1}(\psi)}{c_{2}(\psi)} c_{1}(\phi) \leq & \frac{\mu_{F+\psi \circ \pi}\left(\pi^{-1}\left[y_{0}, \ldots, y_{n-1}\right]\right)}{m_{\psi}\left(\left[y_{0}, \ldots, y_{n-1}\right]\right)} \\
& \times \exp (n(P(S, F+\psi \circ \pi)-P(T, \psi)-P(S, F+\phi \circ \pi)+P(T, \phi))) \\
\leq & \frac{b_{2}(\psi) c_{2}(\phi)}{c_{1}(\psi)} .
\end{aligned}
$$

So depending on the sign of $P(S, F+\psi \circ \pi)-P(T, \psi)-P(S, F+\phi \circ \pi)+P(T, \phi)$, we have either

$$
\mu_{F+\psi \circ \pi}\left(\pi^{-1}(C)\right) / m_{\psi}(C) \leq b_{2}(\psi) c_{2}(\phi) / c_{1}(\psi)
$$

for all cylinder sets $C$ or

$$
b_{1}(\psi) c_{1}(\phi) / c_{2}(\psi) \leq \mu_{F+\psi \circ \pi}\left(\pi^{-1}(C)\right) / m_{\psi}(C)
$$

for all cylinder sets $C$. In the first case we have $\mu_{F+\psi \circ \pi} \circ \pi^{-1} \ll m_{\psi}$, and in the second case $m_{\psi} \ll \mu_{F+\psi \circ \pi} \circ \pi^{-1}$; and since both measures are ergodic, they must be equal. Therefore,

$$
P(S, F+\psi \circ \pi)-P(T, \psi)=P(S, F+\phi \circ \pi)-P(T, \phi) \quad \forall \psi \in \mathcal{F}(Y),
$$

and therefore for all $\psi \in C(Y)$. If we let $c=P(T, \phi)-P(S, F+\phi \circ \pi)$, then $c+F$ is a compensation function.

We have now proved the equivalence of (i), (ii), (iii) and (iv). Since (v) implies (iv), it will suffice to show that (i) implies (v). This follows from Corollary 3.4.

From (iv) we see that if we know that the unique measure with maximal entropy for $T, m_{0}$, lifts to a measure $\mu$ which is the equilibrium state of some $F \in \mathcal{F}(X)$, then $F+c$ is a compensation function for some $c \in R$.

Notice that when we know $F \in \mathcal{F}(X)$ is a compensation function, then the map $m_{\phi} \rightarrow \mu_{F+\phi \circ \pi}$ gives an injection from the class of all measures which are equilibrium state of functions in $\mathcal{F}(Y)$ to the corresponding class for $X$.

In $[\mathbf{B}, \mathbf{T}]$ it was shown that if we know some Markov measure $\mu_{1} \in M(X, S)$ projects to some Markov measure $m_{1} \in M(Y, T)$, then $F=-I_{\mu_{1}}+I_{m_{1}} \circ \pi$ is a 
compensation function. We can see this from Theorem 4.1, because $\mu_{1}$ is the unique equilibrium state for $-I_{\mu_{1}}$ and $m_{1}$ is the unique equilibrium state for $-I_{m_{1}}$, so by (iv) $c-I_{\mu_{1}}+I_{m_{1}} \circ \pi$ is a compensation function for some $c \in R$. Putting $\phi=-I_{m_{1}}$ in $P\left(S, c-I_{\mu_{1}}+I_{m_{1}} \circ \pi+\phi \circ \pi\right)=P(T, \phi)$, we get $c=0$. By applying (iii) we see that for every Markov measure $m \in M(Y, T)$ there is a Markov measure $\mu$ for $S$ which is the unique one such that $-I_{\mu}+I_{m} \circ \pi$ is cohomologous to $-I_{\mu_{1}}+I_{m_{1}} \circ \pi$. Therefore this choice of compensation function gives an injection from the set of Markov measures in $M(Y, T)$ into the set of Markov measures in $M(X, S)$. If there is another Markov measure $\mu_{2}$ with $\mu_{2} \circ \pi^{-1}=m_{1}$, then $-I_{\mu_{2}}+I_{m_{1}} \circ \pi$ is also a compensation function, and for each Markov measure $m \in M(Y, T)$ we obtain a new Markov lift of $m$. So if $m_{1}, m$ are both Markov measures in $M(Y, T)$, there is a natural bijection between the set of Markov measures in $M(X, S)$ that project to $m_{1}$ and the set of Markov measures in $M(X)$ that project to $m$. The same results hold with the classes of Markov measures on $X$ and $Y$ replaced by the classes of equilibrium states of functions in $\mathcal{F}(X)$ and $\mathcal{F}(Y)$. All these observations were made in $[\mathbf{B}, \mathbf{T}]$.

When the map $\pi$ is finite-to-one, we have the following result of Tuncel. (When $S, T$ are topologically mixing subshifts of finite type, then $\pi$ is finite-to-one iff $\pi$ is bounded-to-one.)

PROPOSITION $4.2[\mathrm{~T}]$. Suppose $\pi$ is finite-to-one. For every $\phi \in \mathcal{F}(Y)$ there is exactly one $\mu \in M(X, S)$ with $\mu \circ \pi^{-1}=m_{\phi}$. The measure $\mu$ is the equilibrium state of $\phi \circ \pi$. Hence if $m_{\phi}$ is Markov so is $\mu$.

ProOF. When $\pi$ is finite-to-one, we have $h_{\mu}(S)=h_{\mu \circ \pi^{-1}}(T) \forall \mu \in M(X, S)$, so that $P(S, \phi \circ \pi)=P(T, \phi) \forall \phi \in C(Y)$. Let $\phi \in \mathcal{F}(Y)$. Let $\mu$ be the unique equilibrium state of $\phi \in \pi$. Then clearly $\mu \in \pi^{-1}$ is an equilibrium state of $\phi$, so it equals $m_{\phi}$. If $\nu \in M(X, S)$ and $\nu \circ \pi^{-1}=m_{\phi}$, then

$$
h_{\nu}(S)+\int \phi \circ \pi d \nu=h_{m_{\phi}}(T)+\int \phi d m_{\phi}=P(T, \phi)=P(S, \phi \circ \pi)
$$

so that $\nu=\mu$.

We have the following characterisation.

THEOREM 4.2. The following statements are equivalent.

(i) $\pi$ is a finite-to-one map.

(ii) 0 is a compensation function.

(iii) Every compensation function in $\mathcal{F}(X)$ is cohomologous to 0.

PROOF. (i) $\Rightarrow$ (iii). If $\pi$ is finite-to-one, then $h_{\mu}(S)=h_{\mu \circ \pi^{-1}}(T) \forall \mu \in M(X, S)$, so that $P(S, \phi \circ \pi)=P(T, \phi) \forall \phi \in C(Y)$. If $\mu_{0}, m_{0}$ denote the measures of maximal entropy on $X, Y$, then, by Proposition 4.2, $\mu_{0} \circ \pi^{-1}=m_{0}$, and, moreover, $\mu_{0}$ is the only member of $M(X, S)$ that projects to $m_{0}$. Suppose $F \in \mathcal{F}(X)$ and $F$ is a compensation function. Then the equilibrium state, $\mu_{F}$, for $F$ projects to $m_{0}$, so $\mu_{F}=\mu_{0}$. By Proposition 4.1(iv) $F$ is cohomologous to a constant $c$; but then

$$
P(T, 0)=P(S, F)=P(S, c)=c+P(S, 0)=c+P(T, 0),
$$

so $c=0$. It suffices to show (ii) $\Rightarrow(\mathrm{i})$. But (ii) implies $h(S)=h(T)$, and by a result of Parry and Tuncel $[\mathbf{P}, \mathbf{T}$, p. 56] $\pi$ is finite-to-one. 
We noted in Proposition 4.2 that when $\pi$ is finite-to-one each Markov measure $m$ on $Y$ is covered by a unique Markov measure on $X$. However, when $\pi$ is not a finite-to-one map, Boyle and Tuncel $[\mathbf{B}, \mathbf{T}, \S 7]$ have shown that if a Markov measure $m$ on $Y$ lifts to a Markov measure on $X$, then $m$ lifts to uncountably many Markov measures on $X$. By the discussion after Theorem 4.1 it follows that every Markov measure on $Y$ lifts uncountably many Markov measures on $X$. There are examples, however, where no Markov measure on $Y$ lifts to a Markov measure on $X[\mathbf{M}, \mathbf{P}, \mathbf{W}]$ (see later in this section).

Finite-to-one factor maps are special cases of uniform factor maps. The factor map $\pi$ is called uniform if the unique measure of maximal entropy for $S$ projects by $\pi$ to the unique measure of maximal entropy for $T[\mathbf{B}, \mathbf{T}]$. From Theorem 4.1 it follows that $\pi$ is uniform iff it has a constant for a compensation function. Another equivalent condition is that there exist a constant $c$ such that $\{y \in Y \mid P(S, \pi, 0)(y)=$ $c\}$ is a total probability set for $T$. This follows from Theorem 3.3.

We now consider another condition for $F \in \mathcal{F}(X)$ to be a compensation function. If $r$ is the smallest natural number for which a $q$ exists so that the zeroth coordinate of $\pi(x)$ depends only on $\left(x_{q}, \ldots, x_{q+r-1}\right) \forall x \in X$, then we define a cylinder set in $\pi^{-1}\left(0\left[y_{0}, \ldots, y_{n-1}\right]_{n-1}\right)$ to be a cylinder ${ }_{q}\left[x_{q}, \ldots, x_{q+n+r-2}\right]_{q+n+r-2}$ which is a subset of $\pi^{-1}\left(0\left[y_{0}, \ldots, y_{n-1}\right]_{n-1}\right)$.

THEOREM 4.3. Let $S: X \rightarrow X, T: Y \rightarrow Y$ be topologically mixing subshifts of finite type, and let $\pi: X \rightarrow Y$ be a continuous surjection with $\pi \circ S=T \circ \pi$. Let $F \in \mathcal{F}(X)$. Then $F$ is a compensation function iff there exist constants $\alpha, \beta>0$ such that $\forall n \geq 1 \forall y \in Y$ we have $\alpha \leq \sum_{x \in E_{n}(y)} e^{\left(S_{n} F\right)(x)} \leq \beta$ for all sets $E_{n}(y)$ consisting of one point from each cylinder set in $\pi^{-1}\left({ }_{0}\left[y_{0}, \ldots, y_{n-1}\right]_{n-1}\right)$.

PROOF. In this proof $\left[y_{0}, \ldots, y_{n-1}\right]$ means $0\left[y_{0}, \ldots, y_{n-1}\right]_{n-1}$. If $m_{0} \in M(Y, T)$ is the unique measure with maximal entropy, then by Proposition 4.1 there are positive constants $\gamma_{1}, \gamma_{2}$ with

$$
\gamma_{1} \leq m_{0}\left(\left[y_{0}, \ldots, y_{n-1}\right]\right) e^{-n h(T)} \leq \gamma_{2} \quad \forall n \geq 1, \forall y \in Y .
$$

Similarly there are positive constants $\alpha_{1}, \alpha_{2}$ such that

$$
\alpha_{1} \leq \mu_{F}\left(\left[x_{0}, \ldots, x_{n-1}\right]\right) \exp \left(n P(S, F)-\left(S_{n} F\right)(x)\right) \leq \alpha_{2} \quad \forall n \geq 1, \forall x \in X .
$$

From this we get positive constants $\beta_{1}, \beta_{2}$ such that

$$
\begin{aligned}
\beta_{1} \sum_{x \in E_{n}(y)} e^{\left(S_{n} F\right)(x)} & \leq \mu_{F} \circ \pi^{-1}\left(\left[y_{0}, \ldots, y_{n-1}\right]\right) e^{-n P(S, F)} \\
& \leq \beta_{2} \sum_{x \in E_{n}(y)} e^{\left(S_{n} F\right)(x)} \quad \forall n \geq 1, \forall y \in Y .
\end{aligned}
$$

If $F$ is a compensation function, then $\mu_{F} \circ \pi^{-1}=m_{0}$ and $P(S, F)=h(T)$, so we have

$$
\frac{\gamma_{1}}{\beta_{2}} \leq \sum_{x \in E_{n}(y)} e^{\left(S_{n} F\right)(x)} \leq \frac{\gamma_{2}}{\beta_{1}} .
$$

Conversely, suppose $F \in \mathcal{F}(X)$ satisfies the condition stated in the theorem. Let $m_{0} \in M(Y, T)$ be the measure of maximal entropy. Inequalities (1) and (2) hold. From (2) we get

$$
\alpha \beta_{1} \leq \mu_{F} \circ \pi^{-1}\left(\left[y_{0}, \ldots, y_{n-1}\right]\right) e^{-n P(S, F)} \leq \beta_{2} \beta
$$


From this and (1) we have

$$
\begin{aligned}
\frac{\alpha \beta_{1}}{\gamma_{2}} e^{n(P(S, F)-h(T))} & \leq \frac{\mu_{F} \circ \pi^{-1}\left(\left[y_{0}, \ldots, y_{n-1}\right]\right)}{m_{0}\left(\left[y_{0}, \ldots, y_{n-1}\right]\right)} \\
& \leq \frac{\beta \beta_{2}}{\gamma_{1}} e^{n(P(S, F)-h(T))}
\end{aligned}
$$

According to whether $P(S, F) \geq h(T)$ or $P(S, F) \leq h(T)$, we have $m_{0} \ll \mu_{F}$ 。 $\pi^{-1}$ or $\mu_{F} \circ \pi^{-1} \ll m_{0}$. Since both measures are ergodic, we have $m_{0}=\mu_{F} \circ \pi^{-1}$. By Theorem 4.1(iv), $F+c$ is a compensation function for some $c \in R$. From (3) we get $P(S, F)=h(T)$, so that $c=0$. Hence $F$ is a compensation function.

We now consider conditions for the existence of a compensation function in $\mathcal{F}(Y)$.

THEOREM 4.4. For $G \in \mathcal{F}(Y)$ statements (iii), (iv), (v) of Theorem 4.1 (with $F$ replaced by $G \circ \pi)$ are equivalent to the following statements.

(iii) $\forall \psi \in \mathcal{F}(Y)$ we have $\mu_{\psi \circ \pi} \circ \pi^{-1}=m_{\psi-G}$.

(iv) $\exists \psi \in \mathcal{F}(Y)$ with $\mu_{\psi \circ \pi} \circ \pi^{-1}=m_{\psi-G}$.

$(\mathrm{v})^{\prime} \forall \psi \in \mathcal{F}(Y) \mu_{\psi \circ \pi}$ is the unique measure of maximal relative entropy over $m_{\psi-G}$.

The proofs are accomplished by putting $\phi=\psi-G$ in Theorem 4.1.

From (iv)' we see that if the unique measure of maximal entropy, $\mu_{0}$, for $S$ projects to the equilibrium state of some function $H \in \mathcal{F}(Y)$, then for some $c \in R$ the function $c-H \circ \pi$ is a compensation function.

When a compensation function in $\mathcal{F}(Y)$ exists, this does not imply that the unique equilibrium state of an arbitrary $f \in \mathcal{F}(X)$ pushes down to the equilibrium state of a member of $\mathcal{F}(Y)$. For example, let $X=Y=\{0,1\}^{Z}$, and let $\pi: X \rightarrow Y$ be given by defining $\pi(x)=y$, where $y_{n}=x_{n}+x_{n+1} \forall n \in Z$. Then $\pi$ is a two-to-one map, and so $F=0$ is a compensation function. Let $0<p<\frac{1}{2}$. The $(p, 1-p)$-product measure on $X$ and the $(1-p, p)$-product measure on $X$ push down to the same measure $m$ on $Y$. The measure $m$ cannot be the equilibrium state of a member of $\mathcal{F}(Y)$ because such a measure has a unique lift, by Proposition 4.2.

We shall use $\left|\pi^{-1}\left({ }_{0}\left[y_{0}, \ldots, y_{n-1}\right]_{n-1}\right)\right|$ to denote the number of cylinder sets in $\pi^{-1}\left(0\left[y_{0}, \ldots, y_{n-1}\right]_{n-1}\right)$. (See the discussion before Theorem 4.3.)

THEOREM 4.5. Let $S: X \rightarrow X, T: Y \rightarrow Y$ be topologically mixing subshifts of finite type and let $\pi: X \rightarrow Y$ be a continuous surjection with $\pi \circ S=T \circ \pi$. Let $G \in \mathcal{F}(Y)$. Then $G \circ \pi$ is a compensation function iff there exist constants $\alpha, \beta>0$ such that $\forall n \geq 1, \forall y \in Y$,

$$
\alpha \leq e^{\left(T_{n} G\right)(y)}\left|\pi^{-1}\left(0\left[y_{0}, \ldots, y_{n-1}\right]_{n-1}\right)\right| \leq \beta .
$$

This result follows directly from Theorem 4.3.

Consider the following classes of codes (factor maps) between subshifts of finite type: (i) finite-to-one codes (i.e., zero is a compensation function); (ii) uniform codes (i.e., a constant is a compensation function); (iii) codes with a compensation function in $\mathcal{F}(Y)$; (iv) codes with a compensation function in $\mathcal{F}(X)$; (v) codes that do not have a compensation function in $\mathcal{F}(X)$. The first three classes are each subsets of the next class, and class (v) consists of the codes not in class (iv).

An example of a code in class (ii) but not class (i) is when $X=Y \times Y, S=T \times T$, and $\pi: Y \times Y \rightarrow Y$ is projection onto the first component, $\pi(y, z)=y$. The constant $-h(T)$ is a compensation function. 
The following result is useful in calculating compensation functions for subshifts. It gives a formula for relative pressure when $S$ and $T$ are subshifts, not necessarily of finite type.

THEOREM 4.6. Let $S: X \rightarrow X, T: Y \rightarrow Y$ be subshifts, and let $\pi: X \rightarrow Y$ be a continuous surjection with $\pi S=T \pi$. For each $n \geq 1$ and $y \in Y$ let $D_{n}(y)$ consist of one point from each nonempty set $\pi^{-1}(y) \cap_{0}\left[i_{0}, \ldots, i_{n-1}\right]_{n-1}$. Then $\forall f \in C(X)$,

$$
P(S, \pi, f)(y)=\limsup _{n \rightarrow \infty} \frac{1}{n} \log \left(\sum_{x \in D_{n}(y)} \exp \left(\left(S_{n} f\right)(x)\right)\right) .
$$

Proof. Suppose $y \in Y$ and let the sets $D_{n}(y)$ be chosen. Let $\alpha$ denote the natural partition of $X$ into the sets $0[i]_{0}$. Let $\alpha_{k}$ denote $\bigvee_{j=-k}^{k} S^{-j} \alpha$. Suppose, for the moment, that $f$ is a one-sided function. If $a$ is the number of elements in the alphabet of $X$, we have

$$
\begin{aligned}
q_{n}\left(S, \pi, f, \alpha_{k}\right)(y) & \leq a^{k} \sum_{x \in D_{n+k}(y)} \exp \left(\left(S_{n} f\right)(x)\right) \\
& \leq a^{k} e^{k\|f\|} \sum_{x \in D_{n+k}(y)} \exp \left(\left(S_{n+k} f\right)(x)\right) .
\end{aligned}
$$

Proposition 1.3 then gives

$$
P(S, \pi, f)(y) \leq \limsup _{n \rightarrow \infty} \frac{1}{n} \log \left(\sum_{x \in D_{n}(y)} \exp \left(\left(S_{n} f\right)(x)\right)\right) .
$$

Similarly,

$$
\begin{aligned}
p_{n}\left(S, \pi, f, \alpha_{k}\right)(y) & \geq \sum_{x \in D_{n+k}(y)} \exp \left(\left(S_{n} f\right)(x)\right) \\
& \geq e^{-k\|f\|} \sum_{X \in D_{n+k}(y)} \exp \left(\left(S_{n+k} f\right)(x)\right),
\end{aligned}
$$

so that

$$
P(S, \pi, f)(y)=\limsup _{n \rightarrow \infty} \frac{1}{n} \log \left(\sum_{x \in D_{n}(y)} \exp \left(\left(S_{n} f\right)(x)\right)\right) .
$$

The same conclusion holds if $f$ is cohomologous to a one-sided function. If $f(x)$ depends only on a finite number of coordinates of $x$, then $f$ is cohomologous to a one-sided function; since if $f(x)$ depends only on $\left(x_{-k}, \ldots, x_{r}\right)$, then $f(x)=$ $f\left(S^{k} x\right)+\left(-S_{k} f\right)(S x)-\left(-S_{k} f\right)(x)$, and $f \circ S^{k}$ is a one-sided function. Since such functions are dense in $C(X)$, the required formula holds for all $f \in C(X)$.

We now give an example of a code in class (iii) but not in class (ii). Let $X=$ $\left\{a_{1}, a_{2}, b\right\}^{Z}, Y=\{a, b\}^{Z}$, let $S, T$ be the shift maps on $X, Y$, and let $\pi: X \rightarrow Y$ be defined by the one-block map that sends $a_{1}$ and $a_{2}$ to $a$ and sends $b$ to $b$. Suppose $\psi \in C(Y)$. By Theorem 4.6 we have

$$
\begin{aligned}
P(S, \pi, \psi)(y) & =\limsup _{n \rightarrow \infty} \frac{1}{n}\left[\left(T_{n} \psi\right)(y)+\log \left|\pi^{-1}\left(0\left[y_{0}, \ldots, y_{n-1}\right]_{n-1}\right)\right|\right] \\
& =\limsup _{n \rightarrow \infty} \frac{1}{n}\left[\left(T_{n} \psi\right)(y)+(\log 2)\left(T_{n} \chi_{0}[a]_{0}\right)(y)\right] .
\end{aligned}
$$


If $m \in M(Y, T)$, we have

$$
\int P(S, \pi, \psi) d m=\int \psi d m+(\log 2) m\left({ }_{0}[a]_{0}\right)
$$

for $m$-almost all $y \in Y$. If $\psi$ is a constant function taking the value $c$, then $\int P(S, \pi, c) d m=c+(\log 2) m\left({ }_{0}[a]_{0}\right)$ varies with $m \in M(Y, T)$, so $c$ is not a compensation function (Theorem 3.3). However, if $\psi=(-\log 2) \chi_{0}[a]_{0}$, we see that $\int P(S, \pi, \psi) d m=0 \forall m \in M(Y, T)$, so that $(-\log 2) \chi_{0}[a]_{0} \in C(Y)$ is a compensation function.

The following is an example of a code in class (iv) but not in class (iii). Let $Y=\{a, b\}^{Z}$, and let $X$ be the subshift of $\left\{a_{1}, a_{2}, b\right\}^{Z}$ determined by allowing the transitions marked on Diagram 1. The map $\pi$ is the one-block map determined by mapping $a_{1}$ and $a_{2}$ to $a$ and $b$ to $b$. We shall use Theorem 4.5 to show that $G \circ \pi$ is not a compensation function for any $G \in \mathcal{F}(Y)$. Suppose $G \in \mathcal{F}(Y)$ and $G \circ \pi$ is a compensation function. There are positive constants, $\alpha, \beta$, such that

$$
\alpha \leq e^{\left(T_{n} G\right)(y)}\left|\pi^{-1}\left({ }_{0}\left[y_{0}, \ldots, y_{n-1}\right]_{n-1}\right)\right| \leq \beta \quad \text { for all } n \geq 1 \text { and all } y \in Y \text {. }
$$

Let $y$ be the point $a^{\infty}$, which has $y_{n}=a \forall n \in Z$.

Then $\alpha \leq e^{n G\left(a^{\infty}\right)}(n+1) \leq \beta$. If $G\left(a^{\infty}\right)>0$, then the middle term goes to $\infty$, if $G\left(a^{\infty}\right)<0$ the middle term goes to zero, and if $G\left(a^{\infty}\right)=0$ the middle term goes to $\infty$. Since none of these can happen, we know there is no compensation function in $F(Y)$. However, for any $t<0$ the function

$$
F(x)=t \chi_{0}\left[a_{1}, a_{1}\right]_{1}(x)+\log \left(1-e^{t}\right) \chi_{0}\left[a_{2}, a_{1}\right]_{1}(x)
$$

is a compensation function. This follows from Theorem 4.6 and the calculation

$$
\begin{aligned}
e^{k F\left(a_{1}, a_{1}\right)}+\sum_{j=1}^{k} & \exp \left((j-1) F\left(a_{1}, a_{1}\right)+F\left(a_{2}, a_{1}\right)\right) \\
& =e^{k t}+\sum_{j=1}^{k} e^{(j-1) t}\left(1-e^{t}\right)=e^{k t}+\frac{e^{t}-1}{1-e^{t}}\left(e^{k t}-1\right)=1 .
\end{aligned}
$$

An example of a code in class ( $v$ ) is the code of Marcus, Petersen and Williams, which they showed maps no Markov measure on $X$ to a Markov measure on $Y$ $[\mathbf{M}, \mathbf{P}, \mathbf{W}]$. Here the space $Y$ is the subset of $\{a, b\}^{Z}$ defined by the allowable transitions given in Diagram 2: The space $X$ has 24 symbols, and the allowable transitions are given in Diagram 3, where only the symbols $b, a_{1}, a_{1}^{\prime}, a_{2}, a_{2}^{\prime}, a_{2}^{\prime \prime}$ are given names and the other symbols are represented by dots.

The map $\pi: X \rightarrow Y$ is the one-block map defined by sending $b$ to $b$ and sending all other symbols for $X$ to the symbol $a$ for $Y$. We shall use $\left[z_{0}, \ldots, z_{n-1}\right]$ to denote the set $0\left[z_{0}, \ldots, z_{n-1}\right]_{n-1}$. The notation $a^{n}$ denotes the string $(a, \ldots, a)$ of length $n$. If $C_{1}, C_{2}, \ldots, C_{N}$ are blocks of symbols, then $\left[C_{1} \cdot C_{2} \cdots C_{N}\right]$ denotes the cylinder set obtained by juxtaposing the blocks in the order shown. If $C$ is a block of length $s$ in $Y$, then $C^{\infty}$ denotes the point $\bigcap_{n=-\infty}^{\infty} T^{-n s} C$ in $Y$. Similarly, for a block in $X$.

We shall use the following three properties of $\pi$ : for any $k \geq 0$

$$
\pi^{-1}\left[b a^{6 k+3} b\right]=\left[b a_{1}\left(a_{1}^{\prime} a_{1}\right)^{3 k+1} b\right] .
$$




$$
\begin{gathered}
\pi^{-1}\left[b a^{6 k+4} b\right]=\left[b a_{2}\left(a_{2}^{\prime} a_{2}^{\prime \prime} a_{2}\right)^{2 k+1} b\right] . \\
\pi^{-1}\left[b a^{6 k+1} b\right]=\left[b a_{1}\left(a_{1}^{\prime} a_{1}\right)^{3 k} b\right] \cup\left[b a_{2}\left(a_{2}^{\prime} a_{2}^{\prime \prime} a_{2}\right)^{2 k} b\right] .
\end{gathered}
$$

For $k \geq 0$ let $C_{1}(k)=\left[b a_{1}\left(a_{1}^{\prime} a_{1}\right)^{3 k+1}\right], C_{2}(k)=\left[b a_{2}\left(a_{2}^{\prime} a_{2}^{\prime \prime} a_{2}\right)^{2 k+1}\right], C_{3}(k)=$ $\left[b a_{1}\left(a_{1}^{\prime} a_{1}\right)^{3 k}\right]$ and $C_{4}(k)=\left[b a_{2}\left(a_{2}^{\prime} a_{2}^{\prime \prime} a_{2}\right)^{2 k}\right]$.

Suppose there is a compensation function $F \in \mathcal{F}(X)$. Since any function cohomologous to $F$ is also a compensation function, we can assume $F$ is a one-sided function satisfying condition (*). By Theorem 4.3 there are positive constants $\alpha, \beta$ such that

$$
\alpha \leq \sum_{x \in E_{n}(y)} \exp \left(\left(S_{n} F\right)(x)\right) \leq \beta \quad \forall n \geq 1, \forall y \in Y
$$

and for all sets $E_{n}(y)$ consisting of one point from each cylinder set in

$$
\pi^{-1}\left({ }_{0}\left[y_{0}, \ldots, y_{n-1}\right]_{n-1}\right) .
$$

If we put $n=N(6 k+4)+1$ and $y=\left[b a^{6 k+3}\right]^{\infty}$ in (4), we get

$$
\alpha \leq \exp \left(N\left[\left(S_{6 k+4} F\right)\left(\left(C_{1}(k)\right)^{\infty}\right)\right]+F\left(\left(C_{1}(k)\right)^{\infty}\right)\right) \leq \beta \quad \forall N \geq 1 .
$$

Therefore

$$
\left(S_{6 k+4} F\right)\left(\left(C_{1}(k)\right)^{\infty}\right)=0
$$

Similarly

$$
\left(S_{6 k+5} F\right)\left(\left(C_{2}(k)\right)^{\infty}\right)=0 .
$$

In a similar way if we choose $j_{i} \in\{1,2\}$ for $1 \leq i \leq N$ and $r$ of them are equal to 2 , then

$$
\left(S_{N(6 k+4)+r} F\right)\left(\left(C_{j_{1}}(k) \cdot C_{j_{2}}(k) \cdots C_{j_{N}}(k)\right)^{\infty}\right)=0 .
$$

If we put $n=N(6 k+2)+1$ and $y=\left[b a^{6 k+1}\right]^{\infty}$ in (4), we get

$$
\begin{gathered}
\alpha \leq \sum_{j_{1}, \ldots, j_{N} \in\{3,4\}} \exp \left[\left(S_{N(6 k+2)} F\right)\left(\left(C_{j_{1}}(k) \cdot C_{j_{2}}(k) \cdots C_{j_{N}}(k)\right)^{\infty}\right)\right] \\
\cdots \exp F\left(\left(C_{j_{1}}(k) \times C_{j_{N}}(k)\right)^{\infty}\right) \leq \beta
\end{gathered}
$$

Therefore

$$
\begin{aligned}
\alpha e^{-\|F\|} & \leq \sum_{j_{1}, \ldots, j_{N} \in\{3,4\}} \exp \left[\left(S_{N(6 k+2)} F\right)\left(\left(C_{j_{1}}(k) \cdot C_{j_{2}}(k) \cdots C_{j_{N}}(k)\right)^{\infty}\right)\right] \\
& \leq \beta e^{\|F\|} \quad \forall N \geq 1 .
\end{aligned}
$$

Let

$$
\begin{aligned}
\omega_{k}(F)=\sup _{n \geq 1}\left[\operatorname { s u p } \left\{\sum_{i=1}^{n} F\left(a_{-i}, \ldots, a_{-1} x^{+}\right)-F\left(a_{-i}, \ldots, a_{-1} z^{+}\right) \mid\right.\right. \\
\left.\left.\left(a_{-n}, \ldots, a_{-1} x^{+}\right) \text {is allowed and }\left(x_{0}, \ldots, x_{k-1}\right)=\left(z_{0}, \ldots, z_{k-1}\right)\right\}\right] .
\end{aligned}
$$

By condition $(*)$ we have $\omega_{k}(F) \rightarrow 0$ as $k \rightarrow \infty$. 
We shall use condition (5) to show $F\left(\left[a_{1}^{\prime} a_{1}\right]^{\infty}\right)+F\left(\left[a_{1} a_{1}^{\prime}\right]^{\infty}\right)=0$. Let $m<k$. Then $0=\left(S_{6 k+4} F\right)\left(\left(C_{1}(k)\right)^{\infty}\right)$ differs from

$$
\begin{aligned}
F\left(\left(C_{1}(k)\right)^{\infty}\right) & +(3 k+1-m) F\left(\left(a_{1} a_{1}^{\prime}\right)^{\infty}\right)+(3 k+1-m) F\left(\left[a_{1}^{\prime} a_{1}\right]^{\infty}\right) \\
& +\sum_{j=1}^{m} F\left(\left[a_{1}^{\prime} a_{1}\right]^{j}\left(C_{1}(k)\right)^{\infty}\right)+\sum_{j=0}^{m-1} F\left(\left[a_{1} a_{1}^{\prime}\right]^{j} a_{1}\left(C_{1}(k)\right)^{\infty}\right)
\end{aligned}
$$

by at most $2 \omega_{2 m}(F)$. Since we can let $k \rightarrow \infty$, we get

$$
F\left(\left[a_{1} a_{1}^{\prime}\right]^{\infty}\right)+F\left(\left[a_{1}^{\prime} a_{1}\right]^{\infty}\right)=0 .
$$

Similarly, using (6), we get

$$
F\left(\left[a_{2}^{\prime} a_{2}^{\prime \prime} a_{2}\right]^{\infty}\right)+F\left(\left[a_{2}^{\prime \prime} a_{2} a_{2}^{\prime}\right]^{\infty}\right)+F\left(\left[a_{2} a_{2}^{\prime} a_{2}^{\prime \prime}\right]^{\infty}\right)=0 .
$$

We now use (8), (9), (10) to get a contradiction to $\omega_{k}(F) \rightarrow 0$.

Consider (8). For each choice of $j_{1}, \ldots, j_{N} \in\{3,4\}$ we shall compare the quantity

$$
\left(S_{N(6 k+2)} F\right)\left(\left(C_{j_{1}}(k) \cdot C_{j_{2}}(k) \cdots C_{j_{N}}(k)\right)^{\infty}\right)
$$

with

$$
\left(S_{N(6 k+4)+r} F\right)\left(\left(C_{j_{1}-2}(k) \cdot C_{j_{2}-2}(k) \cdots C_{j_{N-2}}(k)\right)^{\infty}\right),
$$

where $r$ is the number of $j_{i}$ equal to 4 . The last expression is zero by (7). Suppose $j_{1}=3$. Then

$$
\left(S_{6 k+2} F\right)\left(\left(C_{3}(k) \cdot C_{j_{2}}(k) \cdots C_{j_{N}}(k)\right)^{\infty}\right)
$$

differs from

$$
\left(S_{6 k+4} F\right)\left(\left(C_{1}(k) \cdot C_{j_{2}-2}(k) \cdots C_{j_{N}-2}(k)\right)^{\infty}\right)
$$

by at most $3 \omega_{2 k}(F)$; since

$$
\left(S_{4 k+2} F\right)\left(\left(C_{3}(k) \cdot C_{j_{2}}(k) \cdots C_{j_{N}}(k)\right)^{\infty}\right)
$$

differs from

$$
\left(S_{4 k+2} P\right)\left(\left(C_{1}(k) \cdot C_{j_{2}-2}(k) \cdots C_{j_{N}-2}(k)\right)^{\infty}\right)
$$

by at most $\omega_{2 k}(F)$,

$$
\left(F \circ S^{4 k+2}+F \circ S^{4 k+3}\right)\left(\left(C_{1}(k) \cdot C_{j_{2}-2}(k) \cdots C_{j_{N}-2}(k)\right)^{\infty}\right)
$$

differs from $F\left(\left[a_{1} a_{1}^{\prime}\right]^{\infty}\right)+F\left(\left[a_{1}^{\prime} a_{1}\right]^{\infty}\right)=0$ by at most $\omega_{2 k}(F)$, and

$$
\sum_{i=4 k+2}^{6 k+1} F \circ S^{i}\left(\left(C_{3}(k) \cdot C_{j_{2}}(k) \cdots C_{j_{N}}(k)\right)^{\infty}\right)
$$

differs from

$$
\sum_{s=4 k+4}^{6 k+3} F \circ S^{s}\left(\left(C_{1}(k) \cdot C_{j_{2}-2}(k) \cdots C_{j_{N}-2}(k)\right)^{\infty}\right)
$$

by at most $\omega_{2 k}(F)$.

When $j_{1}=4$ we do a similar argument, using (10) instead of (9) to deal with the extra terms. We then deal with the next $6 k+2$ terms in the sum (11) by considering $C_{j_{2}}(k)$, and continue in this way. We get that expression (11) has absolute value at most $3 N \omega_{2 k}(F)$. Putting this in (8) gives $2^{N} \exp \left(-3 N \omega_{2 k}(F)\right) \leq \beta e^{\|F\|} \forall N, k \geq 1$. Therefore $2 \exp \left(-3 \omega_{2 k}(F)\right) \leq 1$, contradicting the fact that $\omega_{2 k}(F) \rightarrow 0$ as $k \rightarrow \infty$. 
5. An example where no compensation function exists. We now give an example of two topologically transitive homeomorphisms $S: X \rightarrow X, T: Y \rightarrow Y$ and a continuous surjection $\pi: X \rightarrow Y$ with $\pi S=T \pi$ for which no compensation function exists. Both $S$ and $T$ are subshifts of a shift on a countable number of symbols. We first describe the space $X$. Suppose $W=\{0\} \cup\{1 / n\}_{1}^{\infty}$ with the topology inherited from $R$. Let $\Lambda_{1}=\left\{1, \frac{1}{2}\right\}, \Lambda_{2}=\left\{\frac{1}{3}, \frac{1}{4}, \frac{1}{5}\right\}, \Lambda_{3}=\left\{\frac{1}{6}, \frac{1}{7}\right\}, \Lambda_{4}=$ $\left\{\frac{1}{8}, \frac{1}{9} \frac{1}{10}\right\}$, etc. Then $\left\{\Lambda_{n}\right\}$ partitions $\{1 / n \mid n \geq 1\}$, and $\Lambda_{2 n+1}$ has two members, and $\Lambda_{2 n}$ has three members for all $n \geq 1$. Let $X_{n}=\Lambda_{n}^{Z}$, considered as a subset of $W^{Z}$. The closed sets $X_{n}$ are mutually disjoint subsets of $W^{Z}$, and $\bigcup_{n=1}^{\infty} X_{n}$ has closure $\left\{0^{\infty}\right\} \cup \bigcup_{n=1}^{\infty} X_{n}$, where $0^{\infty}$ is the point in $W^{Z}$ whose components are all 0 . Let $U: W^{Z} \rightarrow W^{Z}$ denote the shift map. Then $h\left(\left.U\right|_{X_{2 n}}\right)=\log 3$ and $h\left(\left.U\right|_{X_{2 n+1}}\right)=\log 2$. Let $X_{0}=\left\{0^{\infty}\right\} \cup \bigcup_{n=1}^{\infty} X_{n}$.

We want to find a point $w \in W^{Z}$ so that $X_{0}$ is contained in the closure, $X$, of $\left\{U^{n} z \mid n \in Z\right\}$ and every $\mu \in M(X, U \mid X)$ has $\mu\left(X_{0}\right)=1$. We can do this by making a list consisting of all blocks of zeros, all blocks that occur in $X_{1}$, all blocks that occur in $X_{2}$, etc., and constructing $z=\left(z_{n}\right)_{-\infty}^{\infty}$ by writing down this list of blocks in some order to give the sequence $\left(z_{n}\right)_{-\infty}^{\infty}$. Let $S=U \mid X$. Clearly $X_{0} \subset X$. If $\omega \in X \backslash X_{0}$ and $\omega=\left(\omega_{n}\right)_{-\infty}^{\infty}$ contains a block of symbols $B_{1} \cdot B_{2} \cdot B_{3}$, where $B_{1}$ and $B_{2}$ are blocks from different $X_{n}$ and $B_{2}$ and $B_{3}$ are blocks from different $X_{m}$, then $\omega$ is a wandering point for $S: X \rightarrow X$. The only other possibility for a point $\omega \in X \backslash X_{0}$ is that for some $N$ all the symbols $\omega_{i}$, for $i \leq N$, are symbols from some $X_{n}$, and $\omega_{i}$, for $i>N$, are symbols from some $X_{k}, k \neq n$. If $\Omega(S)$ denotes the nonwandering set of $S$, then such a point cannot belong to $\Omega\left(S \mid \Omega_{(S)}\right)$. Therefore $\mu \in M(X, S)$ implies $\mu\left(X_{0}\right)=1$.

Since $\mu \in M(X, S)$ implies $\mu\left(X_{0}\right)=1$, we have $P(S, f)=P\left(\left.S\right|_{X_{0}},\left.f\right|_{X_{0}}\right)$ by the variational principle. Let $\pi: X \rightarrow W^{Z}$ be defined by mapping the symbol 0 to itself and by mapping each member of $\Lambda_{n}$ to the symbol $1 / n$. Let $Y=\pi(X)$ and $T=U \mid Y$. Hence the image of $X_{n}$ is one point $y^{(n)} \in Y$ and $y^{(n)} \rightarrow 0^{\infty}$. Each point $y^{(n)}$ is a fixed point of $T$. By the same reasoning as above every member of $M(Y, T)$ is concentrated on $\left\{0^{\infty}\right\} \cup\left\{y^{(n)} \mid n \geq 1\right\}$, so that

$$
P(T, \phi)=\max \left(\phi\left(0^{\infty}\right), \sup \left\{\phi\left(y^{(n)}\right) \mid n \geq 1\right\}\right)
$$

Let us suppose there exists $F \in C(X)$ with $P(S, F+\phi \circ \pi)=P(T, \phi) \forall \phi \in C(Y)$. For each $k \geq 1$ and each $b>0$ let $\phi_{k}^{(b)} \in C(Y)$ have the property that $\phi_{k}^{(b)}\left(y^{(n)}\right)=0$ if $n \neq k$ and $\phi_{k}^{(b)}\left(y^{(k)}\right)=b$. Then $P\left(T, \phi_{k}^{(b)}\right)=b$. Therefore

$$
b=P\left(S, F+\phi_{k}^{(b)}\right)=\max \left(F\left(0^{\infty}\right), P\left(S_{k}, F_{k}\right)+b, \sup \left\{P\left(S_{n}, F_{n}\right) \mid n \neq k\right\}\right),
$$

where $S_{n}=\left.S\right|_{X_{n}}$ and $F_{n}=\left.F\right|_{X_{n}}$. By considering large $b$ we see that $P\left(S_{k}, F_{k}\right)=0$. This is true for each $k \geq 1$. Therefore $-\sup F_{k} \leq h\left(S_{k}\right) \leq-\inf F_{k}$, and, using the continuity of $F$, this gives $h\left(S_{k}\right) \rightarrow-F\left(0^{\infty}\right)$. However, $h\left(S_{2 n}\right)=\log 3$ and $h\left(S_{2 n+1}\right)=\log 2$, so $\lim _{k \rightarrow \infty} h\left(S_{k}\right)$ does not exist. This contradiction shows that no compensation function exists. 


\section{REFERENCES}

[A,R] L. M. Abramov and V. A. Rohlin, Entropy of a skew-product transformation with invariant measure, Vestnik Leningrad Univ. 17 (1962), 5-13; English transl., Amer. Math. Soc. Transl. (2)48 (1966), 255-265.

[B] R. Bowen, Equilibrium states and ergodic theory of Anosov diffeomorphisms, Lecture Notes in Math., vol. 470, Springer-Verlag, Berlin, 1975.

[B,T $]$ M. Boyle and S. Tuncel, Infinite-to-one codes and Markov measures, Trans. Amer. Math. Soc. 285 (1984), 657-683.

[H] G. A. Hedlund, Endomorphisms and automorphisms of the shift dynamical system, Math. Systems Theory 3 (1969), 320-375.

[I] R. B. Israel, Convexity in the theory of lattice gasses, Princeton Univ. Press, Princeton, N. J., 1979.

[L] F. Ledrappier, Principe variational et systèmes symboliques, Comment. Math. Phys. 33 (1973), 119-128.

$[\mathbf{L}, \mathbf{W}]$ F. Ledrappier and $\mathrm{P}$. Walters, $A$ relativised variational principle for continuous transformations, J. London Math. Soc. 16 (1977), 568-576.

$[\mathbf{M}, \mathbf{P}, \mathbf{W}]$ B. Marcus, K. Petersen and S. Williams, Transmission rates and factors of Markov chains, Contemp. Math. 26 (1984), pp. 279-293.

[M,P] N. G. Markley and M. E. Paul, Equilibrium states of grid functions, Trans. Amer. Math. Soc. 274 (1982), 169-191.

$[\mathbf{P}, \mathbf{T}]$ W. Parry and S. Tuncel, Classification problems in ergodic theory, London Math. Soc. Lecture Notes, vol. 67, Cambridge Univ. Press, Cambridge, 1982.

[R] D. Ruelle, Thermodynamic formalism, Encyclopedia of Math., Vol. 5, Addison-Wesley, Reading, Mass., 1978.

[T] S. Tuncel, Conditional pressure and coding, Israel J. Math. 39 (1981), 101-112.

[W $\mathbf{W}_{1}$ P. Walters, Ruelle's operator theorem and g-measures, Trans. Amer. Math. Soc. 214 (1975), 375-387.

[ $\left.\mathbf{W}_{\mathbf{2}}\right]$, Invariant measures and equilibrium states for some mappings which expand distances, Trans. Amer. Math. Soc. 236 (1978), 121-153.

[W $\left.\mathbf{W}_{3}\right]$ __ An introduction to ergodic theory, Graduate Texts in Math., no. 79, Springer-Verlag, Berlin, 1982.

MATHEMATICS INSTITUTE, UNIVERSITY OF WARWICK, COVENTRY CV4 7AL, ENGLAND 